ARTICLE

\title{
Structures and function of a tailoring oxidase in complex with a nonribosomal peptide synthetase module
}

Camille Marie Fortinez ${ }^{1,2}$, Kristjan Bloudoff ${ }^{1,2}$, Connor Harrigan ${ }^{1,2}$, Itai Sharon (1D ${ }^{1,2}$, Mike Strauss (D) ${ }^{2,3} \&$

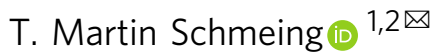

Nonribosomal peptide synthetases (NRPSs) are large modular enzymes that synthesize secondary metabolites and natural product therapeutics. Most NRPS biosynthetic pathways include an NRPS and additional proteins that introduce chemical modifications before, during or after assembly-line synthesis. The bacillamide biosynthetic pathway is a common, threeprotein system, with a decarboxylase that prepares an NRPS substrate, an NRPS, and an oxidase. Here, the pathway is reconstituted in vitro. The oxidase is shown to perform dehydrogenation of the thiazoline in the peptide intermediate while it is covalently attached to the NRPS, as the penultimate step in bacillamide D synthesis. Structural analysis of the oxidase reveals a dimeric, two-lobed architecture with a remnant RiPP recognition element and a dramatic wrapping loop. The oxidase forms a stable complex with the NRPS and dimerizes it. We visualized co-complexes of the oxidase bound to the elongation module of the NRPS using X-ray crystallography and cryo-EM. The three active sites (for adenylation, condensation/cyclization, and oxidation) form an elegant arc to facilitate substrate delivery. The structures enabled a proof-of-principle bioengineering experiment in which the BmdC oxidase domain is embedded into the NRPS.

\footnotetext{
${ }^{1}$ Department of Biochemistry, McGill University, Montréal, QC H3G OB1, Canada. ${ }^{2}$ Centre de recherche en biologie structurale, McGill University, Montréal,

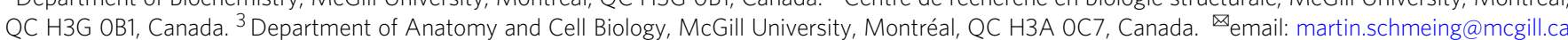


$\mathrm{N}$ onribosomal peptides are a large group of natural products, bioactive secondary metabolites that are often useful to society as therapeutics and green chemicals ${ }^{1,2}$. They include important medicines such as immunosuppressants (rapamycin), antivirals (cyclosporin A), antibiotics (daptomycin), antifungals (caspofungin), and antitumors (bleomycin) ${ }^{3-5}$. Despite their modest size of $\sim 2-18$ residues, nonribosomal peptides exhibit an impressive range of bioactivities because they can occupy a relatively large volume of chemical space. Nonribosomal peptides can contain many residues not typically found in proteins, such as D-, methylated, halogenated, and other nonproteogenic aminoacyl residues, aryl acyl residues, fatty acyl residues, and hydroxy acyl residues. Furthermore, nonribosomal peptides often contain heterocycles or are macrocyclic or branched. These topologies provide advantages such as pre-organization for binding targets or protease resistance ${ }^{6-10}$. The varied, powerful bioactivities and interesting chemical structures of nonribosomal peptides have led to a large number of studies on the total synthesis of the peptides and on biosynthesis and bioengineering of the enzymes which make them ${ }^{11}$.

Nonribosomal peptides are made in microbes by elegant biosynthetic megaenzymes called nonribosomal peptide synthetases (NRPSs) ${ }^{12,13}$. NRPSs are organized as assembly lines of repeating sets of domains. Each set of domains, known as a module, is responsible for adding one acyl monomer substrate, typically an amino acid residue, to the growing peptide chain ${ }^{1}$. A minimal NRPS elongation module contains three domains: the adenylation (A) domain selectively binds the monomer substrate, activates it by adenylation, and transfers it as an aminoacyl thioester to the prosthetic phosphopantetheine (ppant) moiety on the peptidyl carrier protein (PCP) domain. The PCP domain transports the covalently bound amino acid to the condensation (C) domain. The $\mathrm{C}$ domain catalyzes peptide bond formation between the aminoacyl residue on this PCP and the peptidyl moiety on the upstream $\mathrm{PCP}_{\mathrm{n}-1}$ domain, elongating the peptide chain. The PCP domain, now with the newly elongated nascent peptide, translocates to the downstream module, where the peptide is further elongated and passed downstream in the next condensation reaction.

Variations and additions to basic NRPS biosynthesis contribute to the diversity of nonribosomal peptides. These variations can occur before, during, and after NRPS assembly-line synthesis. First, NRPS substrates can be produced from cellular metabolites by dedicated, pre-assembly-line synthetic enzymes ${ }^{14,15}$. Second, tailoring can occur during NRPS assembly-line synthesis, as an additional step in the catalytic cycle of a module. This can be encoded into the NRPS enzyme itself: the NRPS module can contain an alternative tailoring domain such as the heterocyclization (Cy) domain, which replaces the $\mathrm{C}$ domain and performs both condensation and heterocyclization ${ }^{16-18}$, or the NRPS module can include an additional tailoring domain such as a reductase, methylation, monooxygenases, oxidase or formylation domain, which acts on a biosynthetic intermediate tethered to the PCP domain ${ }^{19-21}$. Tailoring during NRPS assembly-line synthesis can also be catalyzed by separately encoded enzymes: Enzymes including halogenases, oxidases, hydroxylase, and P450s ${ }^{22-26} \mathrm{can}$ interact non-covalently with NRPSs and act on PCP-tethered intermediates. Third, after the nonribosomal peptide is released from the NRPS, one or more separately encoded enzymes can catalyze additional reactions to yield the final natural product ${ }^{27}$. Tailoring after release from the NRPS occurs in chloroeremomycin, vancomycin, and penicillin biosynthesis ${ }^{28-30}$.

A full biosynthetic pathway for a nonribosomal peptide can thus involve as few as one enzyme (an NRPS), or may require NRPSs as well as other enzymes for pre-, co-, and/or postassembly-line synthetic steps. Genes for such enzymes are often found together with the NRPS genes in the producer microbe, in a biosynthetic gene cluster (BGC) ${ }^{31}$. However, from the sequence of a BGC it is difficult to predict whether a separately encoded enzyme will act on an acyl substrate before assembly-line synthesis, on a PCP-tethered intermediate of NRPS assemblyline synthesis, or on a small molecule after release from the NRPS. The natures of these possible substrates are quite different, and an enzyme will act on only one of these species $^{24,32}$.

The bacillamide biosynthetic pathway (Fig. 1a) is a threeprotein BGC that contains genes for BmdA, tryptophan decarboxylase which converts L-Trp into tryptamine $(\mathrm{Tpm})^{33}$; $\mathrm{BmdB}$, an NRPS with domains $\mathrm{A}_{1}-\mathrm{PCP}_{1}-\mathrm{Cy}_{2}-\mathrm{A}_{2}-\mathrm{PCP}_{2}-\mathrm{C}_{3}$ that include alternative tailoring domain $\mathrm{Cy}_{2}$, which installs a thiazoline ring in the peptide intermediate ${ }^{16}$; and $\mathrm{BmdC}$, which had not been studied but can be recognized by its sequence as an oxidase enzyme in the nitro-FMN reductase superfamily ${ }^{34,35}$. Mature bacillamides have all been reported to contain thiazole rings $^{36-39}$, and thus BmdC appeared likely to oxidize the thiazoline ring to the thiazole seen in bacillamide D (Fig. 1a), but there is no indication of the timing of this oxidation.

Here, we describe studies on bacillamide synthesis that reveal unexpected biochemical aspects of the system, including stable dimerization induced by a tailoring protein, and an elegant NRPS-tailoring protein structure, previously undescribed for any nonribosomal peptide synthesis.
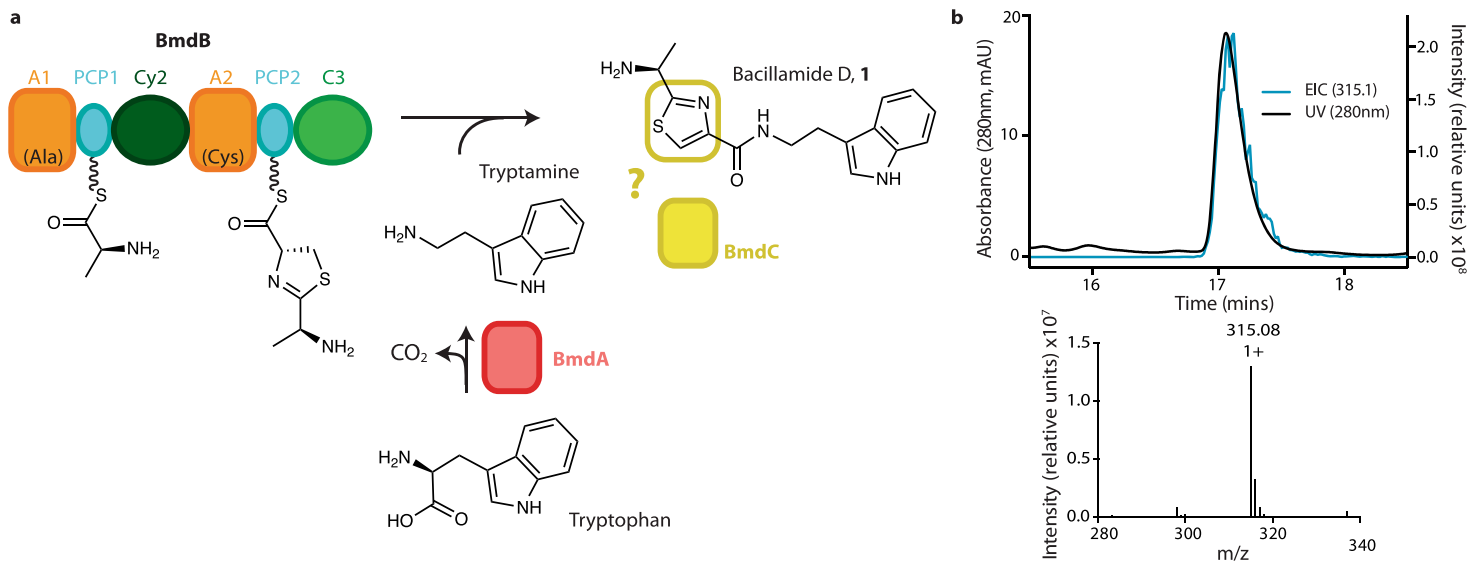

Fig. 1 Biosynthesis of bacillamide D. a A schematic drawing of the biosynthetic pathway of bacillamide D. The domains that make up BmdB are colored orange (adenylation domains), cyan (PCP domains), dark green (heterocyclization (Cy) domain), and green (terminal C domain). BmdC is depicted in yellow and BmdA in red. The color scheme is followed for all figures. $\mathbf{b}$ Mass spectrometry analysis of in vitro reconstitution of the biosynthesis of bacillamide $D$ by BmdA, BmdB, and BmdC. Supplementary Fig. 1 shows the series of bacillamide biosynthesis reactions including controls. 


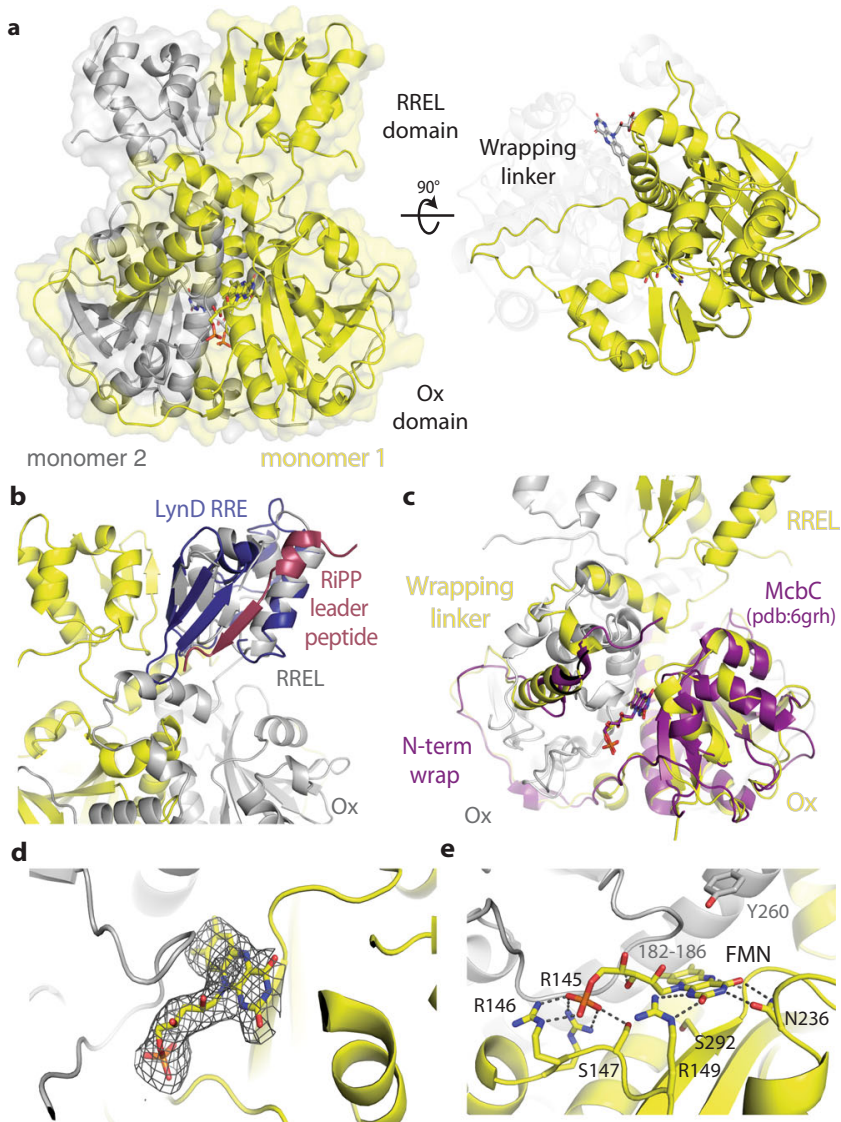

Fig. 2 The structure of the oxidase enzyme BmdC. a BmdC is a dimeric, didomain enzyme. One protomer is shown in yellow and the symmetry mate that completes the biological dimer is shown in gray. $\mathbf{b}$ Superimposition of the RiPP recognition element (RRE) of LynD (blue; PDB code: $4 \mathrm{v} 1 \mathrm{t})^{46}$ on the RREL domain of BmdC. The RiPP leader peptide bound to the LynD RRE is shown in red. c Superimposition of the Ox domain of McbC (purple, PDB: $6 \mathrm{grh})^{42}$ on the Ox domain of BmdC shows the structural similarities including the flavodoxin fold and extended wrapping linker. d Simulated annealing omit $\mathrm{F}_{\mathrm{O}}-\mathrm{F}_{\mathrm{C}}$ electron density map showing binding of $\mathrm{FMN}$ cofactor to BmdC. e The FMN binding site of BmdC.

\section{Results}

In vitro reconstitution of the bacillamide $D$ biosynthetic pathway. The bacillamide BGC is one of the best-represented pathways in genomic databases. Database searches return $>400$ clusters encoding putative proteins with high sequence identity to $\mathrm{BmdA}, \mathrm{BmdB}$, and BmdC, including 350 clusters which show $>65 \%$ protein identity. The clusters are mostly found in Bacilli, though other Bacillaceae are represented, such as Laceyella and Thermoactinomyces species. Yuwen et al. showed B. atrophaeus BmdA is a pyridoxal-5'-phosphate (PLP)-dependent tryptophan decarboxylase $^{33}$, and we showed Thermoactinomyces vulgaris F-5595 BmdB produces pro-bacillamide (AlaCys $s_{\text {thiazoline }} \mathrm{Tpm}$ ) in NRPS assembly-line synthesis ${ }^{16}$ (Fig. 1).

To perform in vitro reconstitution of the full bacillamide $D$ biosynthetic pathway, we heterologously expressed T. vulgaris BmdA, $\mathrm{BmdB}$ and $\mathrm{BmdC}$ in E. coli individually and purified them to homogeneity (Supplementary Fig. 1a). Reactions containing BmdA, $\mathrm{BmdB}$ and BmdC, PLP, ATP, alanine, cysteine, and tryptophan displayed robust production of a species of $\left[\mathrm{M}+\mathrm{H}^{+}\right]=315.14$ (Fig. 1b). This mass and comparison to synthetic standard confirmed this compound as AlaCys thiazole Tpm, i.e., bacillamide D (compound 1) (Supplementary Fig. 1b), indicating that the full bacillamide $D$ pathway was reconstituted in vitro. Reactions which contained tryptamine in place of BmdA, PLP and tryptophan also showed robust bacillamide D production (Supplementary Fig. 1e), indicating that BmdA can fulfill its role simply by producing tryptamine, and that interaction of $\mathrm{BmdA}$ with $\mathrm{BmdB}$ or $\mathrm{BmdC}$ is not required. Removal of BmdC from the reaction conditions gave a species with $\left[\mathrm{M}+\mathrm{H}^{+}\right]=317.15$, identified as AlaCys thiazoline $^{\text {Tpm (pro-bacilla- }}$ mide, compound 2) ${ }^{16}$ (Supplementary Fig. 1f-h). These in vitro reconstitutions confirm $\mathrm{BmdC}$ as the oxidase involved in the bacillamide biosynthetic pathway (Fig. 1).

The structure of the oxidase BmdC. We performed X-ray crystallography of BmdC to gain a better understanding of its structure and function. Highly purified BmdC was subjected to sparse array crystallization. Initial yellow-colored crystals were obtained, consistent with the presence of a flavin cofactor. Iterative optimization gave crystals that yielded high-quality diffraction datasets (Supplementary Table 1). Molecular replacement using a nitro-FMN reductase domain from Anabaena variabilis (PDB ID 3eo7, unpublished) as a search model allowed structure determination at $2.7 \AA$ resolution (Supplementary Table 1, PDB:7ly6). A single copy of BmdC is present in the asymmetric unit (Fig. 2 and Supplementary Fig. 2), and the adjacent symmetrically related copy completes the biological dimer. Nitro-FMN reductase domain protein typically exists as dimers or pseudodimers ${ }^{40}$, and size-exclusion chromatography experiments reveal that $\mathrm{BmdC}$ is dimeric in solution (Supplementary Fig. 2e).

The structure of BmdC shows it to be a dimer of di-domain protomers (Fig. 2 and Supplementary Fig. 2). The small $\mathrm{N}$-terminal domain contains 96 residues and features a winged helix-turn-helix motif (wHTH) with a very small $\beta$-sheet. A $\mathrm{DALI}^{41}$ structural similarity search reveals this domain to be similar to the precursor peptide recognition elements (RRE) in ribosomally synthesized post-translationally modified peptide (RiPP) processing enzymes with $\mathrm{Z}$-scores ranging from 5.3 to 6.5 and rmsd values of 2.0-2.9 $\AA$ over 61-69 alpha carbons (Fig. 2b $)^{42-46}$. These RREs are often found in RiPP pathways for the production of linear azole-containing peptides, azolecontaining cyanobactins and thiopeptides ${ }^{43}$. The larger C-terminal (Ox) domain is comprised of residues 142-325 and folds into a six-stranded $\beta$-sheet sandwiched between $\alpha$-helices on both sides, similarly to the flavodoxin-like fold in other nitroFMN reductases ${ }^{47}$. BmdC contains a 45-residue loop segment between the RRE-like (RREL) and Ox domains, that wraps around the $\mathrm{Ox}$ domain of the other protomer. The loop contributes $1764.3 \AA^{2}$ of the total $5777.3 \AA^{2}$ per-protomer surface area buried by BmdC dimerization ${ }^{48,49}$. These very high values of buried surface area are consistent with our observation that BmdC exists as a dimer in solution. The overall structure of BmdC is most similar to ThcOx (PDB: 5lq4), an FMN-dependent oxidase involved in the production of the cyanobactin patellamide ${ }^{50}$, though ThcOx contains additional copies of the RRE and does not contain the wrapping loop (Supplementary Fig. 2c). McbC (PDB: 6grh), an oxidase involved in microcin B17 biosynthesis ${ }^{42}$, does not contain an N-terminal domain, but does have a similarly dramatic wrapping loop (Fig. 2c).

The electron density maps showed very strong density for flavin mononucleotide (FMN) (Fig. 2d). Because the cofactor was co-purified with BmdC, we confirmed its identity through heat denaturation followed by mass spectrometry, which showed its $\left[\mathrm{M}+\mathrm{H}^{+}\right]$of 457.1 , characteristic of FMN and ruling out FAD (Supplementary Fig. 2d). As with other dimeric flavodoxins, BmdC binds FMN in an active site between its protomers (Fig. 2e). One protomer contributes to most of the side-chain interactions, with both the FMN phosphate, (through Arg145, 

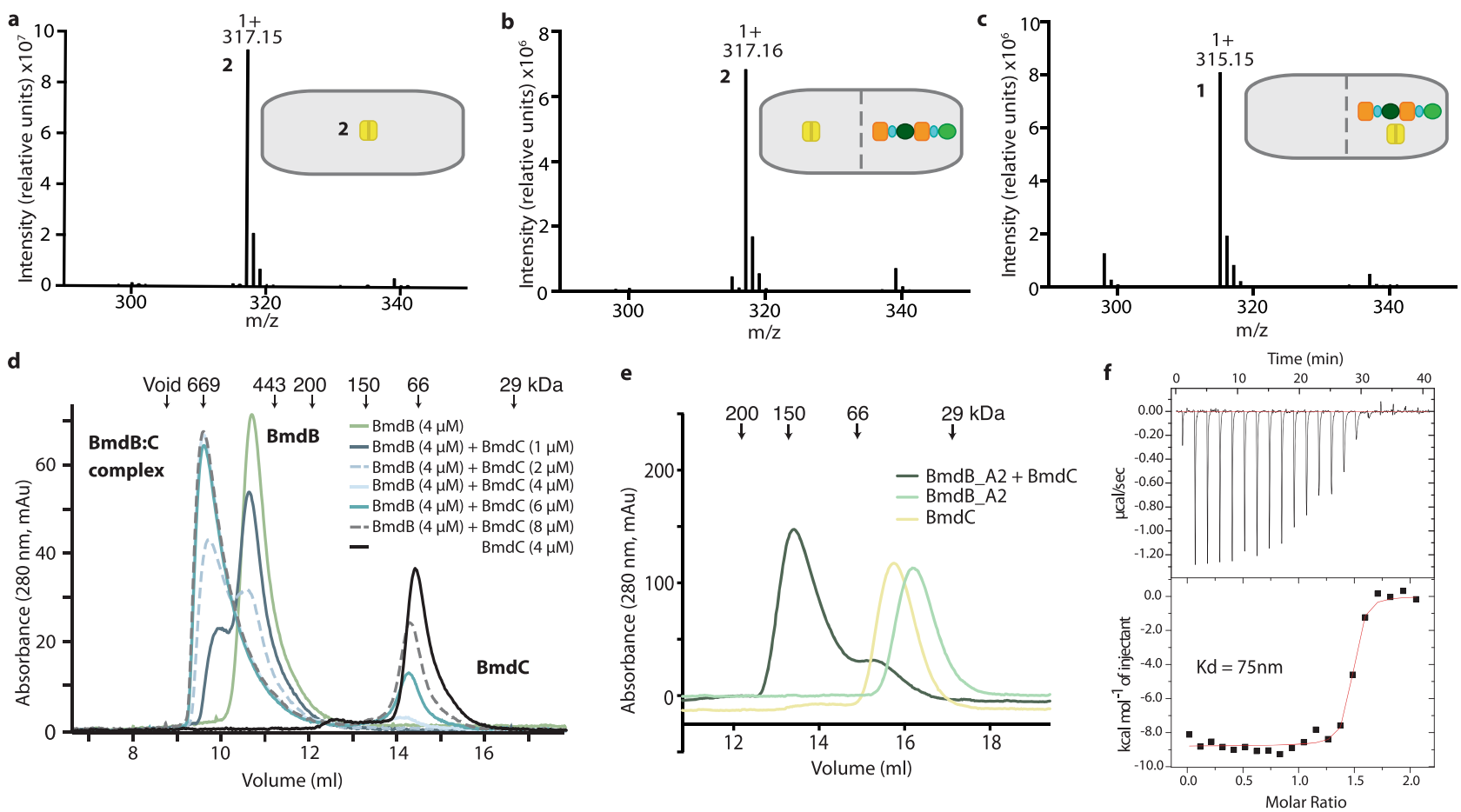

Fig. 3 BmdC must interact with BmdB for oxidation and dimerizes BmdB. a BmdC does not oxidize pro-bacillamide (2), presented as a free small molecule substrate. (Reactions in $\mathbf{a}, \mathbf{b}$, and $\mathbf{c}$ performed in triplicate with similar results.). $\mathbf{b}, \mathbf{c}$ Dialysis experiments show that when BmdC and BmdB are (b) separated by a $10 \mathrm{kDa}$ MW membrane, pro-bacillamide (2) is produced, while when (c) BmdB and BmdC are present on the same side of the membrane, the oxidized bacillamide $D(\mathbf{1})$ is produced. $\mathbf{d}$ Size-exclusion experiments show that the addition of increasing amounts of BmdC lead to the formation of a $B m d B: C$ complex with an elution volume corresponding to binding of two $B m d B$ molecules to a BmdC dimer. e Size-exclusion experiments show $B m d C$ binds to the $A_{2}$ domain of $B m d B$. $f$ Isothermal calorimetry experiments where $A_{2}$ domain of $B m d B$ is titrated by injection into a solution of dimeric $B m d C$ show binding at nanomolar affinity. These experiments were performed in triplicate and with Kd values ranging from $44 \mathrm{nM}$ to $118 \mathrm{nM}$.

Arg146 and Ser147) and the catalytic isoalloxazine rings (hydrogen bonding of Arg149 with FMN N1 and O2, Asn236 with N3 and O4, plus Gly294 with N5; analogous to interactions observed in flavin reductase $\mathrm{P}^{51}$ ). The second protomer contributes backbone interactions through residues 182-186. The isoalloxazine ring system appears planar, but ultra-highresolution would be needed to definitively show planarity ${ }^{51}$. The conserved active-site Tyr260 points toward the FMN in each protomer. The analogous tyrosine in indigoidine synthetase has been proposed as a general base for oxidation, and BmdC is likely to use the same catalytic mechanism ${ }^{52}$. There is room for the thiazole ring to bind between this Tyr260 residue and the catalytic FMN N5 atom; a glycine from the crystallization buffer is observed occupying this site.

BmdC acts during BmdB assembly-line synthesis and induces BmdB dimerization. Inspection of the bacillamide BGC and of the BmdC structure does not clearly indicate whether oxidation by BmdC occurs during assembly-line synthesis or after small molecule release from the NRPS. A reaction mixture of $1 \mu \mathrm{M}$ BmdC and $2 \mathrm{mM}$ pro-bacillamide showed no production of bacillamide D (Fig. 3a), suggesting BmdC does not act on free pro-bacillamide. To test directly whether the oxidation occurs with pro-bacillamide or with AlaCys thiazoline $-\mathrm{S}-\mathrm{BmdB}$ as the substrate for BmdC, we designed dialysis experiments. Using dialysis membrane which allows small molecules but not proteins to pass through, we saw that bacillamide $\mathrm{D}$ production occurs only when BmdB and BmdC are found on the same side of the dialysis membrane, not when they are separated (Fig. 3a-c). When $\mathrm{BmdB}$ and $\mathrm{BmdC}$ are separated, only pro-bacillamide is detected. These results suggest that BmdC acts during NRPS assembly-line synthesis and show that it must physically interact with BmdB for its function.

To investigate the interaction between the oxidase $\mathrm{BmdC}$ and the NRPS BmdB, we performed size-exclusion chromatography titration experiments. Mixing increasing amounts of BmdC with fixed amounts of BmdB led to a distinct peak shift corresponding to a BmdB-C complex (Fig. 3d). Interestingly, the BmdB-C complex elutes from the column substantially earlier than expected for a dimer of BmdC binding to a single NRPS. Rather, it corresponds to $\sim 650 \mathrm{kDa}$, consistent with a complex comprised of two molecules of $\mathrm{BmdB}$ and a dimer of $\mathrm{BmdC}(605 \mathrm{kDa})$. This dimerization is unusual, as NRPS systems usually act as monomers ${ }^{53}$.

Although dimerization in NRPSs is rare, polyketide (PKS) systems are typically dimers ${ }^{54-57}$. Complementation experiments, where each protomer of a dimer has a different inactivating mutation (for example in the conserved serines of the carrier proteins (CP) or the active-site cysteines in ketosynthase domains), have been used to show that the growing polyketide passed from one protomer to the other during synthesis ${ }^{58-60}$. We created mutants of $\mathrm{BmdB}$ that prevent pantetheinylation on $\mathrm{PCP}_{1}$ (BmdB_S792A) and $\mathrm{PCP}_{2}\left(\mathrm{BmdB} \_\right.$S1820A), respectively (Supplementary Fig. 3a, b). Neither BmdB_S792A nor BmdB_S1820A produced pro-bacillamide. Crucially, mixing $\mathrm{BmdC}$ with BmdB_S792A and BmdB_S1820A did not lead to substantial bacillamide production either, indicating that the two $\mathrm{BmdB}$ mutants could not complement each other in the context of a BmdC-induced dimer, and that it is unlikely the nascent peptide is passed between protomers.

We next compared rates of peptide synthesis of probacillamide and bacillamide $\mathrm{D}$ by monomeric and dimeric $\mathrm{BmdB}(-\mathrm{BmdC})$. The reconstitution experiments described above show similar total production of pro-bacillamide by BmdB and 
bacillamide $\mathrm{D}$ by $\mathrm{BmdB}-\mathrm{BmdC}$, but that $\mathrm{BmdB}-\mathrm{BmdC}$ proceeds with around twice the initial rate $\left(4.6 \mathrm{~min}^{-1}\right.$ vs $2.5 \mathrm{~min}^{-1}$; Supplementary Fig. 3a-f). The rate difference is likely because of a preference for a thiazole intermediate over a thiazoline intermediate for condensation with tryptamine at the $\mathrm{C} 3$ domain of BmdB. To discern more directly whether dimerization and/or oxidation has impact on the overall rate of synthesis, we created an FMN-free BmdC. BmdC double mutant R146E S292F does not co-purify with FMN, but still induced dimerization of BmdB. Peptide synthesis reactions showed that BmdC_R146E-S292Finduced dimeric $\mathrm{BmdB}$ produces pro-bacillamide at the same rate as (monomeric) BmdB alone (Supplementary Fig. 3f). Therefore, dimerization does not appear to impart a catalytic advantage to bacillamide synthetase.

To identify the domain of BmdB to which BmdC binds, we made a series of $\mathrm{BmdB}$ truncation constructs and tested their ability to bind BmdC by gel filtration. This showed BmdC binds the $\mathrm{A}$ domain of the second module of $\mathrm{BmdB}\left(\mathrm{BmdB}_{\mathrm{M} 2}\right)$ (Fig. $3 \mathrm{e}$ and Supplementary Fig. 4). We confirmed this interaction by isothermal titration calorimetry (Fig. 3f).

Structural investigations of the $\mathrm{BmdB}_{\mathrm{M}_{2}}-\mathrm{BmdC}$ complex. The complex of $\mathrm{BmdB}_{\mathrm{M} 2}-\mathrm{BmdC}$ was subjected to structural investigation by X-ray crystallography and cryo-electron microscopy. $\mathrm{BmdB}_{\mathrm{M} 2}$ was expressed, purified, modified with various substrate analogues, bound with BmdC, and the complex purified by gel filtration prior to crystallization screening and/or grid preparation. We present three resulting structures: $\mathrm{BmdB}_{\mathrm{M} 2}-\mathrm{BmdC}$ complex structure with a cysteine-vinylsulfonamide adenylate ${ }^{61,62}$, determined by X-ray crystallography at $3.8 \AA$ resolution (PDB:7ly7), a $\mathrm{BmdB}_{\mathrm{M} 2}-\mathrm{BmdC}$ complex structure determined by cryo-EM at $3.8 \AA$ resolution (PDB:7ly4), and an in situ proteolyzed complex of two-thirds of the $\mathrm{BmdB} \mathrm{A}_{2}$ domain with the Ox domain of $\mathrm{BmdC}$, determined by X-ray crystallography at $2.5 \AA$ resolution (PDB:7ly5) (Fig. 4, Supplementary Fig. 5, Supplementary Fig. 6, and Supplementary Tables 1-3).

$\mathrm{BmdB}_{\mathrm{M} 2}-\mathrm{BmdC}$ complex is a very elongated dimeric structure in which the two BmdB modules are kept far apart, and in which the active sites accessed by $\mathrm{PCP}_{2}$ form a catalytic arc (Fig. 4). The center of the complex is $\mathrm{BmdC}$ in the same dimeric configuration described above. The area of the BmdC Ox domain most distal from the dimeric interface (residues 223-231 and 303-306) makes a small interface with one end of the A domain (residues $1321-1323,1468,1512-1513)$, burying $491 \AA^{2}$ of surface area per protein. This positions the two $\mathrm{BmdB}_{\mathrm{M} 2}$ modules in a plane perpendicular to the $\mathrm{BmdC}$ rotational axis, stretching out at $\sim 45^{\circ}$ to the core $\mathrm{BmdC}$ dimer interface. The configuration is extremely oblong, with the $\mathrm{BmdB}_{\mathrm{M} 2}$ : $\mathrm{BmdC}$ complex having overall dimensions of $65 \AA$ by $65 \AA$ by $280 \AA$ and placing the $\mathrm{Cy}_{2}$ domains on either side of the massive complex. There is some flexibility in this configuration, with the relative $\mathrm{Ox}: \mathrm{A}_{2}$ orientation varying by $\sim 11^{\circ}$ across the three structures. The $\mathrm{Cy}_{2}: \mathrm{A}_{2}$ interface is largely constant and the distal N-lobe of $\mathrm{Cy}_{2}$ substantially weaker in both EM and crystallography maps, indicative of both N-lobe:C-lobe flexibility 63,64 and overall variation in the periphery of the complex. Furthermore, though both BmdB modules of the $\mathrm{BmdB}_{\mathrm{M} 2}-\mathrm{BmdC}$ complex are visible in EM maps, the highest quality map was obtained by performing refinement with a mask that enveloped the $\mathrm{BmdC}$ dimer and a single $\mathrm{BmdB}_{\mathrm{M} 2}$ protomer, because the modest flexibility of the Ox: $\mathrm{A}_{2}$ interface does break the overall twofold symmetry of the full complex. Notably, the cryo-EM map was calculated using a sample in which the PCP domain was not locked into a single conformation, and as a result, the PCP and $\mathrm{A}_{\text {sub }}$ domains are not visible, despite attempts at focused classification around PCP-binding sites. The EM maps do confirm the overall conformation and mode of dimerization observed by crystallography. In the $\mathrm{BmdB}_{\mathrm{M} 2}-\mathrm{BmdC}$ crystal structure, the cysteine-vinylsulfonamide-adenylate biases the PCP toward the thiolation state ${ }^{62,65}$, and $\mathrm{PCP}_{2}$ is visible in this position (Fig. 4a) with the domain-domain interaction dominated by the A domain helix of residues $1520-1532$ and the PCP helix of residues 1820-1833. Surprisingly, $A_{2 \text { sub }}$ is disordered, despite no visible impediment to its binding in its classic thiolation position ${ }^{66}$, suggesting $A_{2 s u b}$ may not be required to help position $\mathrm{PCP}_{2}$ for aminoacylation.

In the catalytic cycle of module $2, \mathrm{PCP}_{2}$ must visit $\mathrm{A}_{2}$ for thiolation/aminoacylation to ligate the cysteine substrate (forming Cys-S-PCP ${ }_{2}$ ), then the $\mathrm{Cy}_{2}$ domain for condensation and heterocyclization (forming AlaCys thiazoline $-S-\mathrm{PCP}_{2}$ ) and the BmdC Ox domain for oxidation (forming AlaCys thiazole $-S-\mathrm{PCP}_{2}$ ). The positions of the active sites are marked out by: the present BmdB-C crystal structure (step 1-thiolation), previous $\mathrm{Cy}_{2}$ and LgrA $\mathrm{PCP}_{1}-\mathrm{C}_{2}$ containing structures ${ }^{16,17,67}$ ) (steps 2 and $3-$ condensation and heterocyclization), and the position of FMN in BmdC (step 4-oxidation). These three active sites form an inplane arc, with each opening facing the inside of the concave surface (Fig. $4 \mathrm{c}$ ). This decreases the distance $\mathrm{PCP}_{2}$ is required to travel to transport its intermediates.

The proteolyzed complex provides a high-resolution view of the core BmdB-BmdC interface (Fig. 4d). The BmdB-BmdC binding interface is quite small, burying $491 \AA^{2}$ of surface area per molecule in the full proteins. Of this, $423 \AA^{2}$ is present in the truncated complex ${ }^{48,49}$. Isothermal calorimetry binding experiments between $\mathrm{A}_{2}$ and dimeric $\mathrm{BmdC}$ reveal a $\mathrm{K}_{\mathrm{d}}$ value of $\sim 75 \mathrm{nM}$ (Fig. 3f ${ }^{68}$. The interaction features a network of hydrogen bonding, including two central salt bridges, between BmdC Asp225and BmdB Arg1513 and between BmdC Asp231 and BmdB Arg1512, plus side-chain-backbone and backbonebackbone contacts (Fig. 4c). Single point mutation of $\mathrm{A}_{2}$ (R1512D), $\mathrm{A}_{2}$ (R1513D), BmdC(D225R), and BmdC(D231R) each leads to disruption of the $\mathrm{BmdB}-\mathrm{BmdC}$ complex, as observed by gel filtration (Fig. 4e). Peptide synthesis assays with $\mathrm{BmdC}(\mathrm{D} 225 \mathrm{R})$ and wildtype $\mathrm{BmdB}$ decreased oxidation by $\sim 50 \%$, which confirms the importance of this BmdC-BmdB_A interaction. It is not clear whether the remnant oxidation is because $\mathrm{BmdC}(\mathrm{D} 225 \mathrm{R})$ retains some affinity to $\mathrm{A}_{2}$ of $\mathrm{BmdB}$ (albeit not sufficient to maintain the complex through gel filtration), or is caused by the transient binding of $\mathrm{PCP}_{2}$ to $\mathrm{BmdC}$.

Embedding the BmdC oxidase domain into BmdB. NRPSembedded $\mathrm{Ox}$ domains are thought to have arisen from the genetic insertion of genes for stand-alone Ox enzymes into NRPS genes. That the Ox domain of BmdC has homology with NRPSembedded Ox domains ${ }^{47,52,69}$ led us to ask whether we could replicate evolution and embed $\mathrm{BmdC}$ Ox into $\mathrm{BmdB}$ to turn it into an embedded tailoring domain. Searching the NCBI database of nonredundant proteins returned 40 proteins which include both RREL and Ox domains contained within a larger NRPS or PKS system. Thirteen showed similar insertion points within NPRS A domains and featured an average sequence identity of $\sim 45 \%$ to BmdC. Sequence alignments with $\mathrm{BmdB} \mathrm{A}_{2}$ indicate that the embedded $\mathrm{Ox}$ domains are inserted at a point corresponding to that between $\mathrm{A}_{2}$ loop residues Ser1534 and His1535 (Fig. 5). Notably, this loop is adjacent to the helix that includes BmdB Arg1512 and Arg1513 of the BmdB-BmdC interface. We, therefore, created fusion genes in which BmdC, flanked by linkers observed in embedded RREL-Ox didomains from Paenibacillus tianmuensis ( $\operatorname{Link} P t$ ) or from Bacillus pseudomycoides (LinkBp), is inserted into full-length BmdB after residue 1534. By inserting the BmdC Ox domain without the RREL domain were we able 

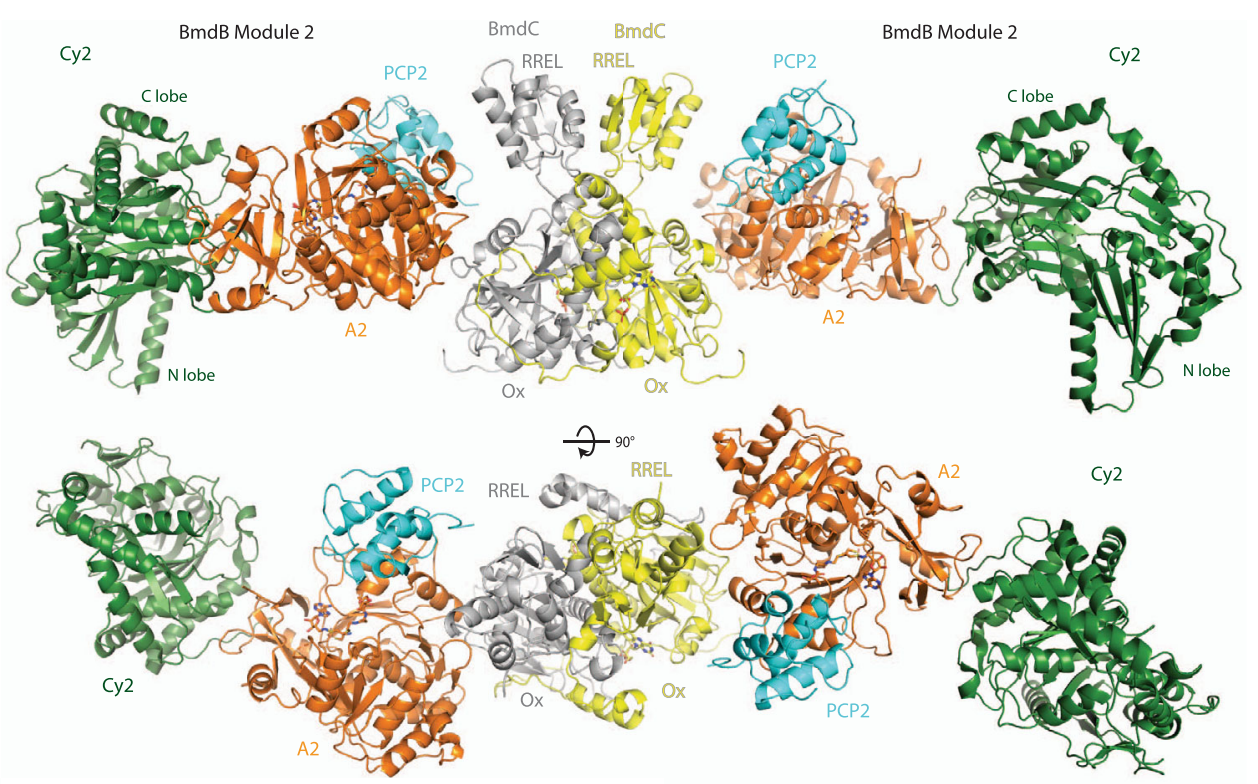

b
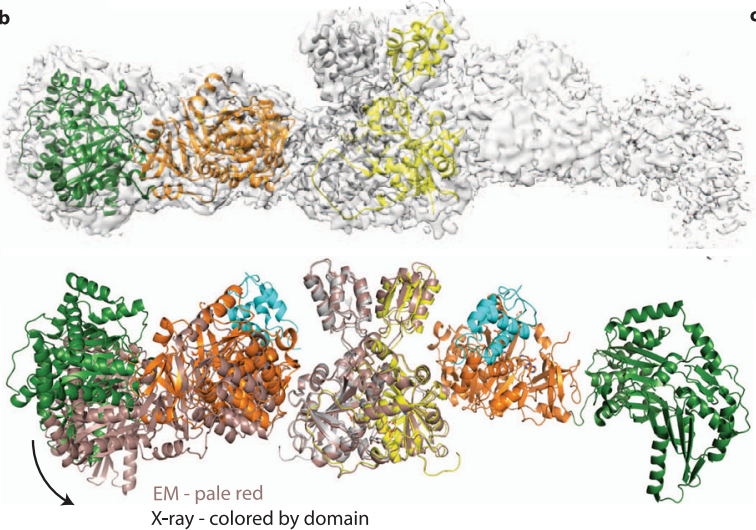

d
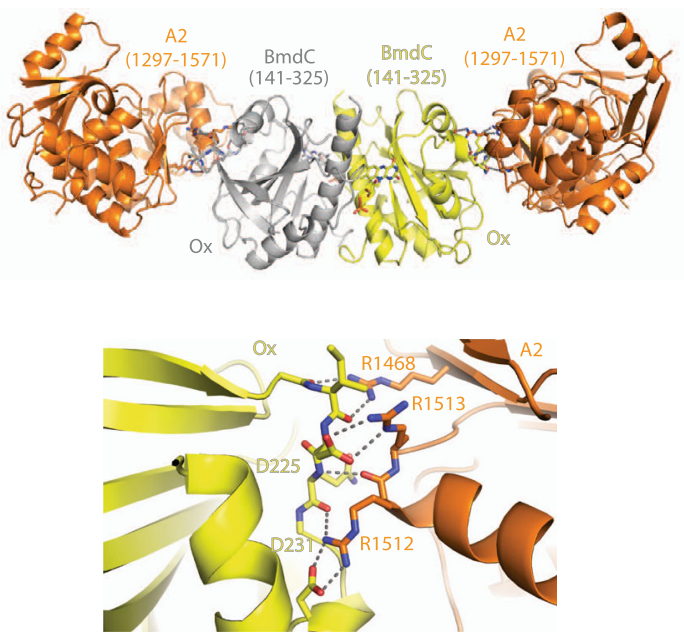

to achieve protein expression and purification. In vitro assays show that these constructs, $\mathrm{BmdB}-\mathrm{BmdC}(\mathrm{Ox}) \operatorname{Link} B p$ and BmdB-BmdC $(\mathrm{Ox}) \mathrm{Link} P t$, are able to produce total bacillamide at $50 \%$ and $25 \%$ efficiency compared to native BmdB-BmdC. Although the total bacillamide produced by $\mathrm{BmdB}-\mathrm{BmdC}(\mathrm{Ox})$ LinkPt is lower, it is more efficient at oxidation, with half the total bacillamide produced being pro-bacillamide and half bacillamide
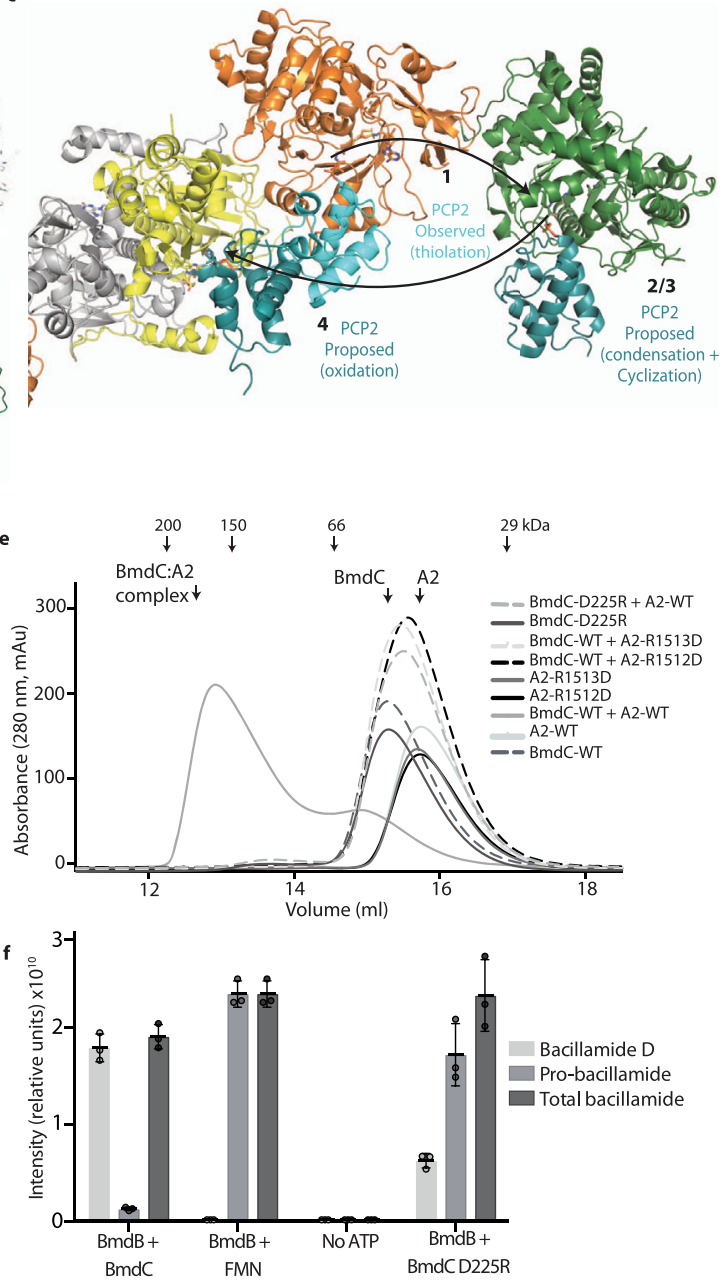

D (Fig. 5). BmdB-BmdC(Ox)_LinkBp oxidizes $\sim 30 \%$ of the bacillamide it produces.

\section{Discussion}

Tailoring domains and proteins play an important part in allowing NRPS products to occupy such a large volume of 


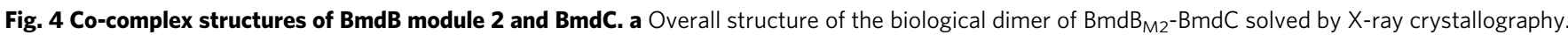
The asymmetric unit contains one protomer each of $\mathrm{BmdB}$ and $\mathrm{BmdC}$ with symmetry mates completing the biological dimers. $\mathbf{b}$ Electron microscopy of the $\mathrm{BmdB}_{\mathrm{M} 2}-\mathrm{BmdC}$. Top: An EM map of the full dimeric $B m \mathrm{mB}_{\mathrm{M} 2}-\mathrm{BmdC}$ (gray) was calculated at $4.2 \AA$ and showed some variability in the relative positions of the $B m d B_{M 2}$. Imposing a mask around the $B m d C$ dimer and one copy of $B m d B$ produced higher resolution maps, at $3.8 \AA$ resolution, into which $B m d C$ and BmdB Cy2A2 could be modeled. Bottom: Superimposition of $B_{m d B}-B m d C$ structures determined by $X$-ray crystallography and by cryo-EM show some modest differences in the BmdC:BmdB interaction angle. $\mathbf{c}$ Proposed positions BmdB PCP2 assumes around the catalytic arc during the synthetic cycle in module 2. The position of PCP2 for thiolation (aminoacylation) (1) is observed in this study, the position for condensation and heterocyclization (2/3) is based on the condensation state of $\operatorname{Lgr} A(P D B: 6 \mathrm{mfz})^{67}$, and the position for oxidation is modeled based on the location of FMN in BmdC. $\mathbf{d}$ The structure of the in situ proteolyzed complex of BmdB_A $A_{2}$ and $B m d C O x$ (top), and a close-up view of the $A_{2}:$ Ox interface (bottom). e Mutations that disrupt either salt bridge in the BmdB:BmdC interface abolished binding between proteins in size-exclusion experiments. Complementing mutations are unable to rescue this interaction. $\mathbf{f}$ Oxidation to bacillamide $\mathrm{D}$ is still possible despite the loss in binding between BmdB and BmdC. The central value for the reactions represents the mean, while the standard deviation of the mean is represented by the error bars. Individual points of the triplicates are shown.

chemical space 28,70 . These domains and proteins are presumably evolutionarily co-opted to function with the NRPS as their genes have been physically taken into the BGC or spliced into the NRPS gene itself. The presence of a RiPP precursor peptide recognitionlike element (RRE) as the $\mathrm{N}$-terminal domain of $\mathrm{BmdC}$ raises the possibility that $\mathrm{BmdC}$ was co-opted from a RiPP cluster into the bacillamide BGC. The RRE binds its RiPP precursor peptide as a beta-strand along the edge of its $\beta$-sheet. This $\beta$-sheet is present in the RRE-like domain of BmdC, and overlaying RRE:peptide complexes shows this beta-strand would point the peptide towards the FMN at the BmdC active site (Fig. 2 and Supplementary Fig. 2b). The heterocyclic peptidyl substrate (Ala-

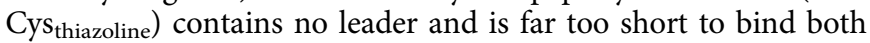
the RRE-like strand and the active site. In addition, the PCP contains no $\beta$ strands, suggesting this RRE feature is likely an interesting evolutionary relic left over from coopting an RiPP oxidase, rather than a functional element in bacillamide synthesis.

The biochemical (Figs. 3 and 4) and structural (Fig. 4) results suggest that oxidation during bacillamide synthesis requires substrate delivery to the $\mathrm{Ox}$ domain by $\mathrm{PCP}_{2}$. It is likely that $\mathrm{Ox}: \mathrm{PCP}_{2}$ protein-protein interactions are required for productive substrate binding to the $\mathrm{Ox}$ active site. The $\mathrm{BmdC}$ structures show the active site of BmdC to be fairly wide and shallow, hinting that a small molecule such as pro-bacillamide may not be able to make extensive binding interactions.

Bacillamide synthetase BmdB exists as a BmdC-induced dimer. As mentioned, dimerization is an uncommon feature in exclusively NRPS systems 53,65 , but is common in polyketide and fatty acid synthases $^{57,58,71}$. One known example of a dimeric NRPS is in the vibriobactin biosynthesis pathway, where NRPS subunit VibF is dimerized through a catalytically inactive $\mathrm{C}$ domain $^{72}$. Similar to the situation with $\mathrm{BmdB}$, this dimerization is not necessary for function and does not significantly increase product formation ${ }^{72}$. Very recently, an elegant structure of a Cy-A-PCP construct of FmoA3 showed it has a head-to-tail homodimer architecture with a massive dimerization interface along the back side of the $\mathrm{Cy}$ and $\mathrm{A}$ domains $^{73}$. The Cy-A domain interface observed in $\mathrm{BmdB}$ is similar to that seen in $\mathrm{FmoA}^{73}$ and in other C-A-containing structures ${ }^{67,74-76}$. The $\mathrm{BmdB}_{\mathrm{M} 2}-\mathrm{BmdC}$ complex structures (Fig. 4) clearly show why dimerization through $\mathrm{BmdC}$ is neutral for bacillamide biosynthesis and why BmdB mutants do not complement each other: The BmdB-BmdC binding interaction holds the $B m d B$ protomers very far apart, such that they are unlikely to interfere with each other. We suggest that dimerization of $B m d B$ through $\mathrm{BmdC}$ is a product of the dimeric nature of $\mathrm{BmdC}$, which is common in oxidases ${ }^{40,51,77,78}$. The twofold symmetry of $\mathrm{BmdC}$ presents two BmdB binding sites that can be occupied simultaneously, and because dimerization does not impart a catalytic disadvantage, there is no evolutionary pressure to eliminate it.

The relatively stable interface between $\mathrm{BmdB} \mathrm{A}_{2}$ and $\mathrm{BmdC}$ means that the flexibility of the $\mathrm{BmdB}_{\mathrm{M} 2}-\mathrm{BmdC}$ interaction is modest. There is clearly some flexibility visible in the EM dataset, so it is more straightforward to refine the region of the map consisting of a single $\mathrm{BmdB}_{\mathrm{M} 2}$ and a dimer of $\mathrm{BmdC}$, but the $\mathrm{BmdB}_{\mathrm{M} 2}$ are in the same general orientation relative to $\mathrm{BmdC}$. This feature of the dimerized module contrasts markedly with the dimodular NRPS linear gramicidin synthetase subunit A, where the first and second modules assume many different relative orientations ${ }^{67}$. The difference is explained by the fact that the two $\mathrm{BmdB}_{\mathrm{M} 2}$ modules are bound to each other through a fairly consistent interaction with the Ox domain of BmdC, whereas modules 1 and 2 of LgrA are tethered by a very flexible linker.

Many separately encoded tailoring enzymes that act during assembly-line synthesis have been described and characterized biochemically ${ }^{23-25}$. However, only systems with P450 tailoring 29,79 and prolyl oxidation ${ }^{80}$ have been structurally characterized, and they feature transient NRPS: tailoring enzymes interactions, rather than the stable tailoring complex reported here. Indeed, although Bmp $3^{80}$ and BmdC are both flavin-dependent oxidases of PCPbound substrates, little other commonality is observed: Bmp3 has a completely different fold from BmdC, uses FAD, not FMN, is tetrameric, and needs to interact only with the type II PCP Bmp1, not with any other NRPS component. The two studies show similar oxidation function can be achieved in very different ways by different NRPS systems.

Oxidation during NRPS assembly-line synthesis can be catalyzed by separately encoded enzymes (in a stable complex like BmdB-BmdC, or a transient complex as in Bmp1:3) and by embedded domains. In addition to the RREL-Ox domains inserted into modules described above, famous examples of thiazole oxidation by embedded domains include those in the biosynthesis of bleomycin, epothilone, and indigoidine ${ }^{69,81-86}$. Bleomycin and epothilone are made by hybrid NRPS-PKS systems, so dimerization of the synthetases is not unexpected. The Ox domains are inserted within the A domain of EpoB and after the PCP domain of BlmIII respectively, and likely contribute to dimerization. As in $\mathrm{EpoB}$, the Ox domain of indigoidine synthetase is inserted between $\mathrm{A}$ domain motifs $\mathrm{A} 8$ and $\mathrm{A} 9$, a common position for tailoring domains: The embedded methyltransferase in TioS is in this position and makes an apparently rigid interface at its insertion site within the $\mathrm{A}_{\text {sub }}$ subdomain ${ }^{87}$. Interestingly, the inserted Ox sequence in indigoidine synthetase (IndC) is larger than that in EpoB (Supplementary Fig. 7b). Careful analysis and structure prediction using the Robetta web server ${ }^{88}$ reveal the insertion is an Ox pseudodimer, suggesting IndC would not dimerize through the inserted pseudodimeric $\mathrm{Ox}$ domain. Indeed, gel filtration of purified IndC shows it is a monomer (Fig. 5). We suggest all indigoidine synthetases will have a pseudodimeric Ox which greatly resembles a BmdC dimer fused to their A domain (Supplementary Fig. 7c).

The in-plane catalytic arc of $\mathrm{BmdB}_{\mathrm{M} 2}: \mathrm{C}$ (Fig. $4 \mathrm{c}$ ) is reminiscent of catalytic chambers in polyketide and fatty acid synthases 89,90 . 
a

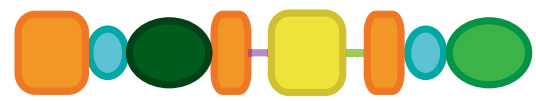

ISSEEQEIGIPTTQR DVIRPEYAagetVRETAAAVTAASGNR Link_PT

SKNELDLREVSIEKSINKQSKIDK KEIESYEEYNQISETENNLKEF Link_BP

b

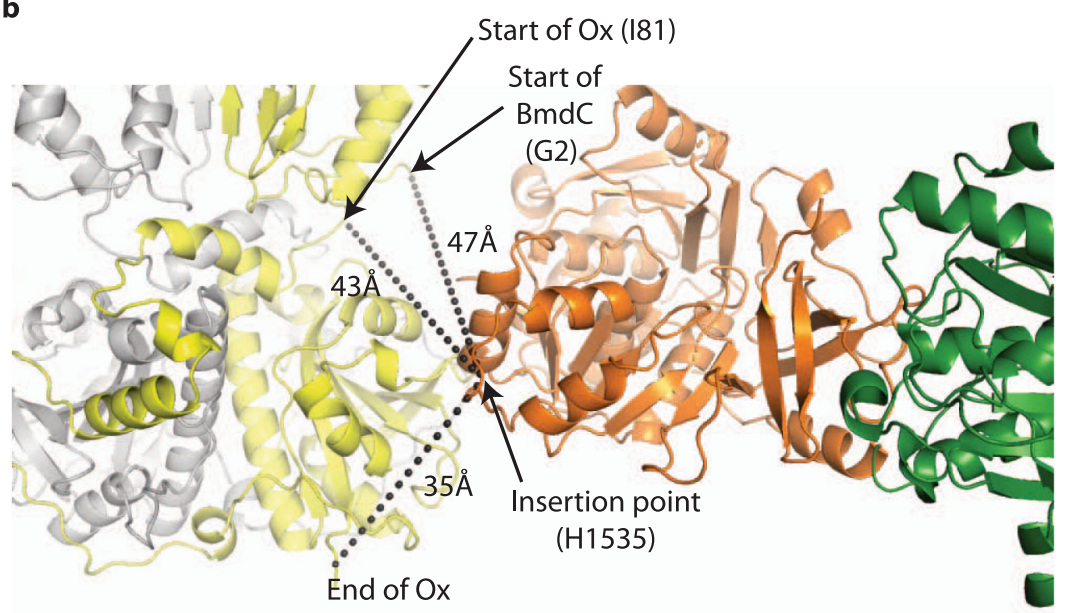

(G325)

c

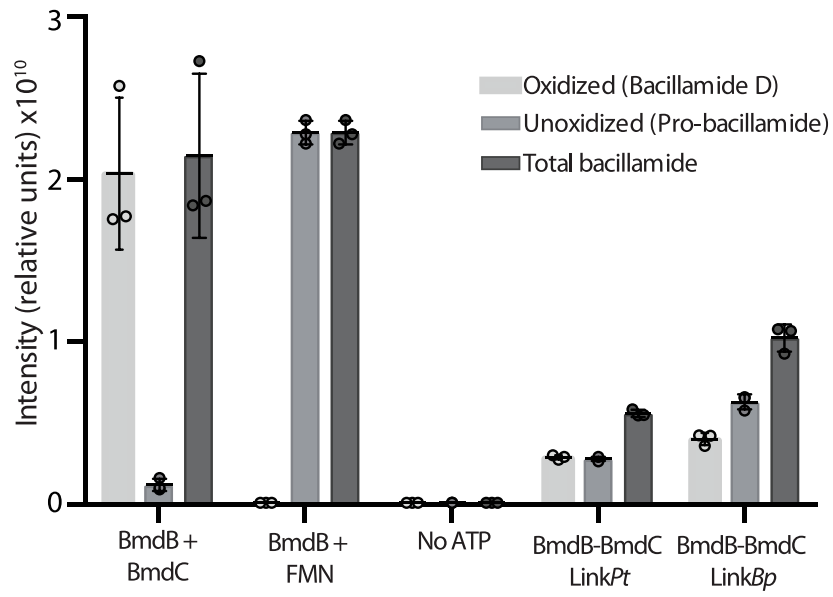

d

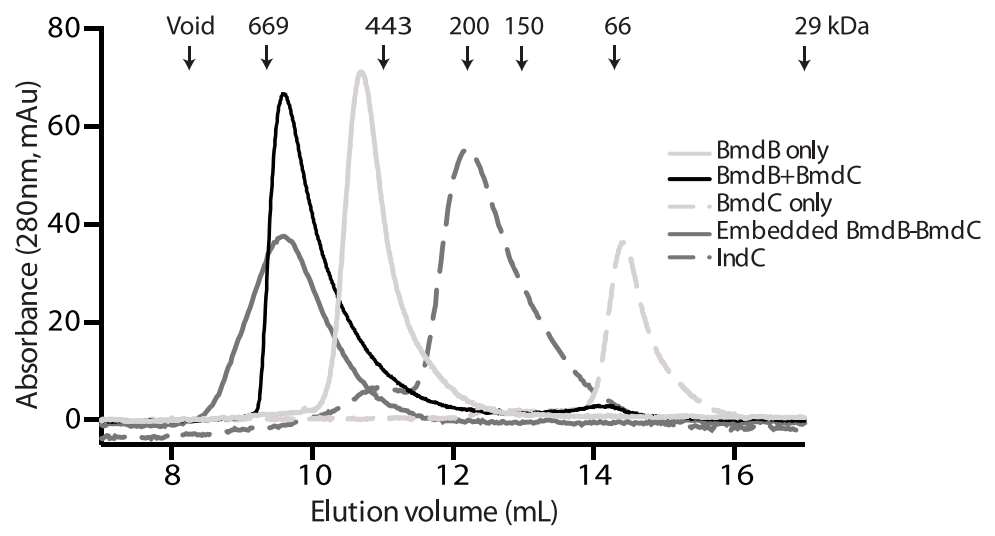

The arc seems to facilitate elegantly the transport function of PCP through the synthetic cycle of module 2 (Fig. 4c), while the BmdB:C geometry keeps each BmdB protomer separate. We believe the BmdB-C complex described here is the only nontransient tailoring complex characterized to date. It remains to be seen whether all such tailoring complexes, which provide a myriad of chemical modifications, have evolved equally elegant architectural solutions.

\section{Methods}

General reagents. All commercial reagents purchased for this study are listed in Supplementary Table 4 and were used without additional purifications.

Cloning and purification of BmdA, B, and C constructs. The proteins used in this study are Thermoactinomyces vulgaris F-5595 BmdA (WP_022737640.1; https:// www.ncbi.nlm.nih.gov/protein/WP_022737640.1?report=genpept), BmdB 
Fig. 5 Embedded Ox domains. a To insert BmdC or the Ox domain of BmdC into BmdB, the linkers with sequences of those found in NRPS with naturally embedded RREL-Ox didomains from Paenibacillus tianmuensis (Link_PT) or from Bacillus pseudomycoides (Link_BP) were used. $\mathbf{b}$ The insertion point of naturally occurring embedded RREL-Ox didomains corresponds to residue H1535 in BmdB. The distances to the beginning of the RREL domain, the beginning of the $\mathrm{Ox}$ and the end of the $\mathrm{Ox}$ domain in BmdC are shown. The number of residues in the chosen linkers is sufficient to span these distances if the linkers are in an extended conformation. $\mathbf{c}$ In vitro peptide synthesis experiments showing the production of bacillamide $D$ and pro-bacillamide in wildtype constructs and the engineered embedded constructs ( $n=3$ independent reactions). The negative control reaction lacks ATP. The central value for the reactions represents the mean. while the standard deviation of the mean is represented by the error bars. $\mathbf{d}$ Gel-filtration experiments show that engineered embedded BmdB-BmdC constructs are dimeric, while IndC is monomeric in solution.

(WP_022737639.1; https://www.ncbi.nlm.nih.gov/protein/WP_022737639.1? report=genpept) ${ }^{16}$ and BmdC (WP_022737638.1; https://www.ncbi.nlm.nih.gov/ protein/WP_022737638.1?report=genpept), cloned from genomic DNA obtained from the Agricultural Research Service Culture Collection. Constructs were cloned into a pET21-derived vector containing an N-terminal TEV-cleavable calmodulinbinding peptide tag and a C-terminal TEV-cleavable octa-histidine tag (pBacTRev), or into a pET21-derived vector containing an $\mathrm{N}$-terminal TEV-cleavable octahistidine tag and a C-terminal TEV-cleavable calmodulin-binding peptide tag (pBacT). All PCR primer sequences, sources of amplified DNA, parental plasmids, restriction sites used, and resulting plasmid names for cloning in this study are listed in Supplementary Table 5. For all site-directed mutagenesis steps, plasmids and primer sequences are listed in Supplementary Table 6.

BmdA was expressed in BL21(DE3) E. coli in LB media containing $40 \mu \mathrm{g} / \mathrm{ml}$ kanamycin at $37^{\circ} \mathrm{C}$. When an $\mathrm{OD}_{600}$ of $\sim 0.5-0.6$ was reached, the cultures were induced with $1 \mathrm{mM}$ of IPTG and further grown at $16^{\circ} \mathrm{C}$ overnight before harvesting by centrifugation. Cell pellets were resuspended in IMAC buffer A (50 mM Tris- $\mathrm{HCl} \mathrm{pH} 7.5,200 \mathrm{mM} \mathrm{NaCl}, 10 \mathrm{mM}$ imidazole $\mathrm{pH}$ 8.0, $2 \mathrm{mM} \beta \mathrm{ME}$ ) and lysed by sonication. The lysate was clarified by centrifugation at $40,000 \times g$ for $20 \mathrm{~min}$, the clarified lysate applied to a $1 \mathrm{ml}$ HiTrap IMAC FF column charged with $\mathrm{Ni}^{2+}$, and protein eluted with IMAC buffer B $(50 \mathrm{mM}$ Tris- $\mathrm{HCl}$ pH 7.5, $200 \mathrm{mM}$ $\mathrm{NaCl}, 250 \mathrm{mM}$ imidazole $\mathrm{pH} 8.0,2 \mathrm{mM} \mathrm{CaCl}, 2 \mathrm{mM} \beta \mathrm{ME}$ ). Fractions containing $\mathrm{BmdA}$ were pooled and applied to a $20 \mathrm{ml}$ calmodulin-binding peptide (CBP) column with equilibrated in CBP buffer A ( $50 \mathrm{mM}$ Tris-HCl pH 7.5, $200 \mathrm{mM}$ $\mathrm{NaCl}, 2 \mathrm{mM} \mathrm{CaCl}, 2 \mathrm{mM} \beta \mathrm{ME})$. BmdA was eluted with CBP buffer B $(50 \mathrm{mM}$ Tris- $\mathrm{HCl} \mathrm{pH} \mathrm{7.5,} 200 \mathrm{mM} \mathrm{NaCl}, 2 \mathrm{mM}$ EGTA pH 8.0, $2 \mathrm{mM} \beta \mathrm{ME}$ ), concentrated and applied to a Superdex200 10/300 column equilibrated GF buffer A plus $1 \mathrm{mM}$ DTT.

BmdC was heterologously expressed with growth, harvest, and lysis as described for BmdA. Clarified lysate was applied to a $5 \mathrm{ml}$ HiTrap IMAC FF column charged with $\mathrm{Ni}^{2+}$, and BmdC eluted with IMAC buffer B. Fractions containing BmdC were pooled and applied to a $20-\mathrm{ml}$ CBP column equilibrated with CBP buffer A, and eluted with $\mathrm{CBP}$ buffer B. Pooled fractions were dialyzed overnight against GF buffer A plus $2 \mathrm{mM} \beta \mathrm{ME}$, at which time affinity tags were cleaved with N-His-TEV protease $\left(1 \mathrm{mg}\right.$ per $20 \mathrm{mg}$ of $\mathrm{BmdC}$ ) at $4{ }^{\circ} \mathrm{C}$. The cleaved $\mathrm{BmdC}$ sample was passed again through the HiTrap IMAC FF and CBP columns, the flowthrough was concentrated and applied to a Superdex 200 16/60 column equilibrated with GF buffer A (50 mM Tris- $\mathrm{HCl} \mathrm{pH} 7.5,200 \mathrm{mM} \mathrm{NaCl}$ ) plus $2 \mathrm{mM} \beta \mathrm{ME}$.

$B m d B$ was expressed and purified similarly to described ${ }^{16}$ : protein was expressed in BL21(Bap1) E. coli ${ }^{91}$, grown in LB media containing $40 \mu \mathrm{g} / \mathrm{ml}$ kanamycin at $37^{\circ} \mathrm{C}$ before being induced, harvested, and purified in the same manner as BmdC.

BmdC_R146E-S292F, BmdC_D225R, BmdC_D231R, and BmdB_C 3 were all expressed and purified as described for BmdC. BmdB $\mathrm{M}_{\mathrm{M} 2}, \mathrm{BmdB}$ _S792A, and BmdB_S1820A were expressed and purified by the same protocol described for BmdC except in BL21(Bap1) E. coli $i^{91}$ and TEV digestion and re-application to affinity columns was not performed. BmdB_A $A_{2}, B m d B \_A_{2}-R 1512 D, B m d B \_A_{2}-$ R1513D and BmdB_PCP 2 were expressed and purified in as described for BmdC, but omitting CBP chromatography.

Bacillamide synthesis assays. Bacillamide synthesis reaction conditions were adapted from Duerfahrt et al. ${ }^{92}$. Reaction containing $1 \mu \mathrm{M}$ BmdA, $1 \mu \mathrm{M}$ BmdB, $1 \mu \mathrm{M}$ BmdC, $1 \mathrm{mM}$ L-alanine, $1 \mathrm{mM}$ L-cysteine, $2 \mathrm{mM}$ ATP, $1 \mathrm{mM}$ pyridoxal-5'phosphate (PLP), $2 \mathrm{mM}$ FMN, $1 \mathrm{mM}$ L-tryptophan, $50 \mathrm{mM}$ HEPES pH 7.5, $100 \mathrm{mM} \mathrm{NaCl}, 0.5 \mathrm{mM}$ TCEP and $10 \mathrm{mM} \mathrm{MgCl}_{2}$ in a final volume of $50 \mu \mathrm{L}$ were incubated for $2 \mathrm{~h}$ at $37^{\circ} \mathrm{C}$. Accompanying reactions lack components/substitute components as indicated in Supplementary Fig. 1 (e.g., no ATP controls; $2 \mathrm{mM}$ tryptamine in place of PLP, tryptophan, and BmdA; no BmdC and FMN reaction). Assays depicted in Supplementary Fig. 1i were performed with $2 \mathrm{mM}$ of probacillamide, $2 \mathrm{mM}$ ATP, $1 \mu \mathrm{M}$ BmdC, $50 \mathrm{mM}$ HEPES pH 7.5, $100 \mathrm{mM} \mathrm{NaCl}$, $0.5 \mathrm{mM}$ TCEP, and $10 \mathrm{mM} \mathrm{MgCl} 2$ in the same volume. Reactions were stopped with $50 \mu \mathrm{L}$ of 4:1 n-butanol:chloroform, lyophilized to dryness and resuspended in $50 \mu \mathrm{L}$ of $10 \%$ methanol. Five microliters of sample were applied to a ZORBAX Extend-C18 (Agilent) column, analyzed with LC-ESI-MS and eluted with LCmethod 1 (Supplementary Table 7). ESI-MS was performed with an in-line Bruker amaZon speed ETD ion-trap mass spectrometer (Fig. 1 and Supplementary Fig. 1). The pro-bacillamide and bacillamide D standards were purchased from Zamboni Chem Solutions. In vitro bacillamide reactions including BmdC_D225R were performed the same way (Fig. 4f). When sets of experiments included quantification of bacillamide $\mathrm{D}$ and pro-bacillamide production (Figs. $4 \mathrm{f}$ and $5 \mathrm{c}$ ), production was quantified using areas under the curves of extracted ion chromatograms (EICs). Bacillamide D was quantified using the area under the EIC for $m / z=315.1$ across the time in which bacillamide peaks elute. Because some samples did not have full separation between peaks for bacillamide D and probacillamide, quantification of pro-bacillamide was performed by integrating the area under the EIC for $m / z=317.1$ and subtracting $6 \%$ of the area under the EIC for $m / z=315.1$, to compensate for the $m / z=317.1$ contribution from isotopic distribution of heavier isotopes within bacillamide $\mathrm{D}$.

BmdC crystallography. Crystals of BmdC formed with the vapor diffusion, sittingwell technique at $22^{\circ} \mathrm{C}$ with a protein concentration of $5 \mathrm{mg} / \mathrm{ml}$ and a crystallization solution of 35\% PEG1500 (wt/vol), 0.1 M SPG buffer ( $0.148 \%$ (w/vol) succinic acid, $0.604 \%(\mathrm{w} / \mathrm{vol})$ sodium dihydrogen phosphate monohydrate, $0.328 \%$ (w/vol) glycine, $\mathrm{pH}$ 9.0). Crystals were cryoprotected by the addition of $10 \%$ ethylene glycol, then looped and flash cooled in liquid nitrogen before data collection. Diffraction data were collected at the CLS 08-ID beamline of the CMCF at the Canadian Light Source $(\lambda=0.979 \AA)$, in Saskatoon, Canada. The data were indexed in the space group $\mathrm{I}_{1} 3$ with iMosflm ${ }^{93}$ and scaled with AIMLESS in $\mathrm{CCP}^{94}$. Initial phases were obtained by molecular replacement using the program MORDA in CCP $4^{95}$ and search models homologous to the Ox domain, including 3EO7 (unpublished). The RREL portion was modeled using AutoBuild in PHENIX $^{96}$. Geometry restraints for FMN were obtained with SKETCHER in CCP $4^{95}$. The overall structure was then iteratively rebuilt in COOT $^{97}$ and refined in PHENIX ${ }^{96}$ to produce the final structure (Supplementary Table 1). There is one molecule in the asymmetric unit, and a symmetry mate forms the biological dimer of BmdC.

Cofactor identification. $\mathrm{BmdC}$ was heat-denatured at $95^{\circ} \mathrm{C}$ for $15 \mathrm{~min}$ and pelleted by centrifugation at $21,100 \times g$ (15,000 RPM with FA-45-24-11 rotor). The supernatant was filtered through a $10000 \mathrm{Da}$ Amicon filtration device and the flowthrough used for HPLC ${ }^{98}$ : Samples were applied on the ZORBAX Extend-C18 column and eluted with LC-method 2 (Supplementary Table 7). The cofactor eluted at $\sim 10 \mathrm{~min}$ and was analyzed by direct injection and ESI-MS with an amaZon speed ETD ion-trap mass spectrometer. The standards of FAD and FMN used were purchased from Sigma-Aldrich.

Dialysis experiments. For dialysis experiments, $1 \mu \mathrm{M}$ BmdB and $1 \mu \mathrm{M} \mathrm{BmdC}$ were placed on either the same side or different sides of dialysis membrane in a Slide-A-Lyzer (10,000 MWCO; Thermo Scientific), in reactions of $400 \mu \mathrm{L}$ of $50 \mathrm{mM}$ Tris- $\mathrm{HCl}$ pH 7.5, $100 \mathrm{mM} \mathrm{NaCl}, 10 \mathrm{mM} \mathrm{MgCl}, 2 \mathrm{mM}$ FMN, $1 \mathrm{mM} \mathrm{L}$ alanine, $1 \mathrm{mM}$ L-cysteine, $10 \mathrm{mM}$ tryptamine, and $2 \mathrm{mM}$ ATP. The reactions proceeded for $2 \mathrm{hrs}$ at $37^{\circ} \mathrm{C}$ with $70 \mathrm{RPM}$ of shaking, and were stopped with $200 \mu \mathrm{L}$ of 4:1 n-butanol:chloroform. Samples were then lyophilized overnight before resuspension in $100 \mu \mathrm{L}$ of $10 \%$ methanol. Samples $(20 \mu \mathrm{L})$ were applied to a ZORBAX Extend-C18 (Agilent) column and eluted with LC-method 3 (Supplementary Table 7). ESI-MS was performed with a Bruker amaZon speed ETD iontrap mass spectrometer.

Gel-filtration binding experiments. Full-length $\mathrm{BmdB}$ and $\mathrm{BmdC}$ at concentration of $1-8 \mu \mathrm{M}$ were mixed and applied to a Superdex200 Increase 10/300 column equilibrated with GF buffer A plus $1 \mathrm{mM}$ DTT (Fig. 3e). To identify the domain of BmdB which binds BmdC (Supplementary Fig. 4), $4 \mu \mathrm{M} \mathrm{BmdB}_{\mathrm{M} 2}, \mathrm{BmdB}_{-} \mathrm{A}_{2} \mathrm{PCP}_{2}$, BmdB_Cy ${ }_{2}{ }^{16}$, BmdB_A $A_{2}$, BmdB_PCP ${ }_{2}$, or BmdB_C $C_{3}$ were mixed with $4 \mu \mathrm{M}$ of BmdC and applied to a Superdex 200 Increase 10/300 column equilibrated with GF buffer A plus $0.5 \mathrm{mM}$ TCEP. For site-directed mutagenesis validation of the BmdB-BmdC binding interface by gel filtration (Fig. 4 e), $5 \mu \mathrm{M}$ of $\mathrm{BmdB} \_\mathrm{A}_{2}$, BmdB_A $A_{2}-R 1512 D$, or BmdB_A $A_{2}-R 1513 D$ was incubated with $5 \mu \mathrm{M}$ of BmdC, BmdC-D225R or BmdC-D231R and applied to the Superdex200 Increase 10/300 column equilibrated with GF buffer A plus $1 \mathrm{mM}$ DTT.

Bacillamide complementation experiments. Complementation experiment reactions included $50 \mathrm{mM}$ HEPES $\mathrm{pH} 7.5,100 \mathrm{mM} \mathrm{NaCl}, 2 \mathrm{mM}$ DTT, $10 \mathrm{mM}$ $\mathrm{MgCl}_{2}, 1 \mathrm{mM}$ L-alanine, $1 \mathrm{mM}$ L-cysteine, $2 \mathrm{mM}$ ATP, $10 \mathrm{mM}$ tryptamine, $2 \mathrm{mM}$ 
FMN, $1 \mu \mathrm{M}$ enzyme in a volume of $200 \mu \mathrm{L}$. Reactions were stopped with $400 \mu \mathrm{L}$ of 4:1 n-butanol:chloroform before lyophilizing overnight and resuspending in $100 \mu \mathrm{L}$ of $10 \%$ methanol for LC-MS analysis. Ten microlitres of the sample was applied to a ZORBAX Extend-C18 (Agilent) column and analyzed with LC-ESI-MS using LCmethod 3 .

Assays with flavin-free BmdC. Each reaction of $500 \mu \mathrm{L}$ had $50 \mu \mathrm{L}$ samples removed at $0,1,2,5,10,15,30,60$, and $120 \mathrm{~min}$. The reaction contained $50 \mathrm{mM}$ HEPES $\mathrm{pH}$ 7.5, $10 \mathrm{mM} \mathrm{MgCl} 2,100 \mathrm{mM} \mathrm{NaCl}, 2 \mathrm{mM}$ DTT, $1 \mu \mathrm{M}$ enzyme(s), $1 \mathrm{mM}$ L-alanine, $1 \mathrm{mM}$ L-cysteine, $2 \mathrm{mM} \mathrm{ATP}, 10 \mathrm{mM}$ tryptamine, plus $2 \mathrm{mM}$ of FMN for the wildtype $\mathrm{BmdC}$ and $\mathrm{BmdB}$ reaction. FMN was not added to the BmdC R146E_S292F because this mutant retains some affinity to FMN, but the $2 \mathrm{mM}$ of FMN was confirmed not to promote oxidation (Supplementary Fig. 1). Reactions were stopped with $50 \mu \mathrm{L}$ 4:1 n-butanol:chloroform before lyophilizing overnight and resuspending in $50 \mu \mathrm{L}$ of $10 \%$ methanol before LC-MS analysis. The sample was centrifuged at $21,100 \times g$ (15,000 RPM with FA-45-24-11 rotor) before applying $10 \mu \mathrm{L}$ of the supernatant to a ZORBAX Extend-C18 (Agilent) column and analyzing with LC-method 3.

Initial rates were estimated by performing a linear regression analysis (with GraphPad Prism version 6.0.0) from 0-30 min intersecting at $y=0$, and converting from $\mu \mathrm{M} / \mathrm{min}$ to moles of bacillamide produced per mole of bacillamide synthetase per minute. The apparent initial rate of bacillamide syntheses are BmdB: $2.5 \mathrm{~min}^{-1}$; BmdB-BmdC: $4.6 \mathrm{~min}^{-1}$; BmdB-BmdC_R146E_S292F: $2.3 \mathrm{~min}^{-1}$.

ITC experiments. Samples were dialyzed overnight in a $1 \mathrm{~L}$ solution of GF buffer A plus $1 \mathrm{mM}$ TCEP at $4{ }^{\circ} \mathrm{C}$. The protein concentrations were measured using the NanoDrop 2000 spectrophotometer (Thermo Scientific) and extinction coefficients of $87140 \mathrm{M}^{-1} \mathrm{~cm}^{-1}$ for the dimeric BmdC and $69010 \mathrm{M}^{-1} \mathrm{~cm}^{-1}$ for BmdB_A $\mathrm{A}_{2}$. ITC was performed with a MicroCal iTC200 (GE Healthcare) with a stirring speed of 750 RPM, $5 \mu$ cals of reference power applied to the reference cell and $2 \mu \mathrm{L}$ injections interspaced by $120 \mathrm{~s}$ in $200 \mu \mathrm{L}$ of sample at $25^{\circ} \mathrm{C}$. The first injection of $0.4 \mu \mathrm{L}$ was removed from the downstream analysis with the software package Microcal Origin 7.0 (OriginLab, Northhampton, MA). Analysis of the titration curves of these experiments was performed with a binding model using a stoichiometry of 1 . The ITC experiment between wildtype BmdC and BmdB $\mathrm{A}_{2}$ was performed by injecting $600 \mu \mathrm{M}$ of BmdB $A_{2}$ into $60 \mu \mathrm{M}$ of dimeric BmdC. These experiments were done in triplicate.

Crystallization of the proteolyzed BmdB-BmdC complex. The proteolyzed $\mathrm{BmdB}-\mathrm{BmdC}$ complex is a result of unexplained proteolysis of both BmdB$\mathrm{A}_{2} \mathrm{PCP}_{2}$ and $\mathrm{BmdC}$ during crystallization. $\mathrm{BmdB} \_\mathrm{A}_{2} \mathrm{PCP}_{2}$ was expressed and purified as described for $\mathrm{BmdB}_{-} \mathrm{A}_{2}$, but adding an anion exchange step before gel filtration, using a Mono Q HR 16/10 column equilibrated in a Q buffer A and a gradient to Q buffer B over $100 \mathrm{~mL}$. The eluted sample was concentrated and applied to a Superdex200 column equilibrated with GF buffer A with $1 \mathrm{mM}$ TCEP.

BmdB_ $\mathrm{A}_{2} \mathrm{PCP}_{2}$ was post-translationally modified using AlaCys ${ }_{\text {thiazole }}$-amino-

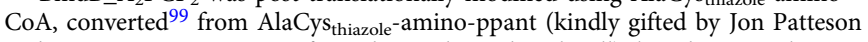
and Dr. Bo Li-University of North Carolina Chapel Hill), but the proteolysis removed the $\mathrm{PCP}_{2}$ domain from the crystallized complex. $\mathrm{A}_{2} \mathrm{PCP}_{2}$ was incubated with a twofold molar excess of BmdC and applied to Superdex 200 Increase 10/300 column with GF buffer B (50 mM Tris- $\mathrm{HCl} \mathrm{pH} \mathrm{7.5,150} \mathrm{mM} \mathrm{NaCl)} \mathrm{with} 1 \mathrm{mM}$ TCEP. Crystals were formed by mixing $4.5 \mathrm{mg} / \mathrm{ml}$ of complex with a crystallization solution of $50 \mathrm{mM}$ Tris- $\mathrm{HCl} \mathrm{pH} \mathrm{7.5,160} \mathrm{mM} \mathrm{KCl,} \mathrm{and} \mathrm{21 \%} \mathrm{PEG3350} \mathrm{(wt/vol),}$ using sitting-drop vapor diffusion at $22^{\circ} \mathrm{C}$. The crystals appeared after $\sim 12$ days and only appear in 24 -well sitting drops with drop ratios of $2 \mu \mathrm{L}$ of protein and $1 \mu \mathrm{L}$ of mother liquor. Crystals were cryoprotected by dipping the crystal in a solution containing $160 \mathrm{mM} \mathrm{KCl}, 21 \%$ PEG3350 (wt/vol) and 20\% ethylene glycol before flash vitrification in liquid nitrogen. Diffraction data were collected using the 24-ID-E beamline of the NE-CAT at the Advanced Photon Source (APS) in Argonne, Illinois. The data were indexed and scaled with HKL2000 ${ }^{100}$ in space group H32. The phases were obtained by molecular replacement in PHENIX ${ }^{96}$ using one chain of BmdC and a homology model of the BmdB $\mathrm{A}_{2}$ domain generated by SWISS-MODEL ${ }^{101}$ as search models. The overall structure was then iteratively rebuilt with COOT $^{97}$ and refined with PHENIX ${ }^{96}$. There is one molecule in the asymmetric unit and a symmetry mate forms the biological dimer of the proteolyzed complex of $\mathrm{BmdB}$ and $\mathrm{BmdC}$.

Crystallization experiments with $\mathbf{B m d B}_{\mathbf{M 2}}-\mathbf{B m d C}$ complex. $\mathrm{BmdB}_{\mathrm{M} 2}$ was heterologously expressed for structure determination from pBacT_BmdB_M2_struct in BL21(EntD-) E. coli ${ }^{102}$ in LB-kan media. $\mathrm{BmdB}_{\mathrm{M} 2}$ was purified as described for $\mathrm{BmdC}$, but adding an anion exchange step before gel filtration, using a Mono $\mathrm{Q} H \mathrm{HR}$ $16 / 10$ column equilibrated in a $\mathrm{Q}$ buffer A (50 mM Tris- $\mathrm{HCl} \mathrm{pH} 7.5,2 \mathrm{mM} \beta \mathrm{ME}$ ) and a gradient to Q buffer B (a Q buffer A plus $1 \mathrm{M} \mathrm{NaCl}$ ) over $150 \mathrm{~mL}$. The eluted sample was concentrated and applied to a Superdex 200 column equilibrated with GF buffer A with $1 \mathrm{mM}$ TCEP.

The purified $\mathrm{BmdB}_{\mathrm{M} 2}$ protein was first modified with ppant from coenzyme A (BioShop Canada Inc.) with $1 \mu \mathrm{M}$ SFP, $10 \mathrm{mM} \mathrm{MgCl}_{2}$, and a twofold molar excess of coenzyme A for $1 \mathrm{~h}$. To remove the excess SFP and coenzyme A, the sample was applied to a Superdex 200 Increase 10/300 column equilibrated with GF buffer B with $1 \mathrm{mM}$ TCEP. Cysteine-vinylsulfonamide adenylate inhibitor (Zamboni Chem Solutions) was used to lock $\mathrm{PCP}_{2}$ in the thiolation state in a reaction with $10 \mathrm{mM}$ $\mathrm{MgCl}_{2}$ and a threefold molar excess of the inhibitor at room temperature overnight. This reaction was then applied to the Superdex 200 Increase 10/300 column equilibrated with the same buffer to separate the excess inhibitor. $\mathrm{BmdB}_{\mathrm{M} 2}$ was incubated with twofold molar excess of purified BmdC and applied to the Superdex 200 Increase 10/300 column equilibrated with GF buffer B with 1 mM TCEP. $\mathrm{BmdB}_{\mathrm{M} 2}: \mathrm{BmdC}$ was crystallized by sitting-drop vapor diffusion after 7 days at $22^{\circ} \mathrm{C}$ using $2 \mu \mathrm{L}$ of $5.6 \mathrm{mg} / \mathrm{ml}$ protein complex and $1 \mu \mathrm{L}$ of crystallization solution (0.2 M sodium citrate, $14 \%$ PEG3350, $5.6 \mathrm{mg} / \mathrm{ml}, 0.08 \mathrm{M}$ guanidine hydrochloride and $0.1 \mathrm{M}$ Bis Tris propane $\mathrm{pH}$ 6.1). Cryoprotection was accomplished by increasing the amount of MPD, using $2 \%$ steps, to a final concentration of $10 \%$, before flash cooling in liquid nitrogen. Diffraction data from this crystal was collected at the CLS 08-ID beamline of the CMCF at the Canadian Light Source $(\lambda=0.979 \AA)$, in Saskatoon, Canada. The data was indexed with iMosflm ${ }^{93}$ to P3 and scaled with AIMLESS to $\mathrm{P}_{5} 22$ in CCP4 $4^{94}$. Initial phases were obtained by molecular replacement in PHENIX ${ }^{96}$ using one copy of BmdC, one copy of the $\mathrm{A}_{2}$ domain fragment solved of the proteolyzed complex structure and one copy of BmdB_Cy $y_{2}$ (PDB: 5t3e) as search models. Geometry restraints for the cysteinevinylsulfonamide adenylate inhibitor were generated with a the combined used of SKETCHER in CCP4 ${ }^{94}$ and REEL in PHENIX ${ }^{96}$. A homology model of the $\mathrm{PCP}_{2}$ domain was generated using SWISS-MODEL ${ }^{101}$ and manually placed in the electron density. The overall structure was then iteratively rebuilt with $\mathrm{COOT}^{97}$ and refined in PHENIX ${ }^{96}$ to lead to the final structure described in Supplementary Table 1 (PDB:7ly7). There is one molecule in the asymmetric unit and a symmetry mate forms the biological dimer of this complex.

BmdB-BmdC(Ox)LinkPt and BmdB-BmdC(Ox)LinkBp constructs and assays. Sequences of linkers used for the BmdB-BmdCLinkPt construct were obtained from an RREL_Ox-embedded NRPS in Paenibacillus tianmuensis (WP_090670142.1-https://www.ncbi.nlm.nih.gov/protein/WP_090670142.1? report=genpept; N-terminal linker ISSEEQEIGIPTTQR; C-terminal linker DVKRPEYAAGETVRETAAAVTAASGNR), and those for BmdB-BmdCLink $B p$ were obtained from an RREL_Ox-embedded NRPS in Bacillus pseudomycoides (WP_081621455.1-https://www.ncbi.nlm.nih.gov/protein/WP_081621455.1? report=genpept; $\mathrm{N}$-terminal linker SKNELDLREVSIEKSINKQSKIDK; C-terminal linker KEIESYEEYNQISETENNLKEF). pBacTRev_BmdB was modified by sitedirected mutagenesis to insert BamH1 and HindIII sites after the codon for His 1535 to make pBacBmdB_BamH1HindIII with primers listed in Supplementary Table 6. Synthetic (Bio Basic Corp) BmdCLinkPt, and BmdCLinkBp sequences in pUC19 were digested by BamH1 and HindIII and ligated into pBacBmdB BamH1HindIII. These initial constructs contain the full-length BmdC. The RREL domains of BmdC within these constructs were removed by site-directed mutagenesis primers listed in Supplementary Table 6 to create BmdB-BmdC(Ox)LinkPt and BmdB-BmdC(Ox)LinkBp construct. These mutants were expressed and purified as described for BmdB_S792A.

Reactions performed with these embedded were in a volume of $100 \mu \mathrm{L}$ of $1 \mu \mathrm{M}$ BmdB construct, $50 \mathrm{mM}$ HEPES $\mathrm{pH} 7.5,100 \mathrm{mM} \mathrm{NaCl}, 0.5 \mathrm{mM}$ TCEP, $10 \mathrm{~m} \mathrm{M}$ $\mathrm{MgCl}_{2}, 1 \mathrm{mM}$ L-alanine, $1 \mathrm{mM}$ L-cysteine, $2 \mathrm{mM}$ ATP, $5 \mathrm{mM}$ tryptamine and $2 \mathrm{mM}$ FMN, incubated for $2 \mathrm{hrs}$ at $37^{\circ} \mathrm{C}$. The reaction was stopped by the addition of $100 \mu \mathrm{L}$ of 4:1 n-butanol:chloroform. These samples were then lyophilized overnight before resuspension in $50 \mu \mathrm{L}$ of $10 \%$ methanol. Sample $(10 \mu \mathrm{L})$ was applied to a ZORBAX Extend-C18 (Agilent) column and analyzed with LC-ESI-MS using LCmethod 3. Bacillamide production was quantified as described above for assays, including BmdC_D225R.

IndC purification. The gene encoding IndC ${ }^{69,103}$ from Streptomyces chromofuscus (AFV27434.1), which produces indigoidine and consists of the domains A-OxPCP-Te, was codon-optimized and synthesized by Bio Basic Corp. and subcloned in the vector pBacT, resulting in the vector pBacT_SC_IndC. IndC was expressed and purified as described for BmdB_S792A.

EM structural determination and analysis. BmdB-BmdC complex formation for EM experiments was performed as described above, with pantetheine or AlaCys thiazole-amino-ppant attached to $\mathrm{PCP}_{2}$. Lacey carbon grids (200 mesh, SPI supplies) were glow discharged for $15 \mathrm{~s}$ at $15 \mathrm{~mA}$. Sample ( $3.5 \mu \mathrm{l})$ of were added to the grid surface, blotted for $2 \mathrm{~s}$, and rapidly plunged into liquid ethane, using a Vitrobot (Mark IV, FEI Company). The frozen grids were imaged at $300 \mathrm{kV}$ in a Titan Krios, equipped with a Gatan K3 and Bioquantum (Gatan Inc.) using SerialEM ${ }^{104}$. Each micrograph was acquired in counting mode and consisted of 40 frames to a total fluence of $109 \mathrm{e}^{-} / \AA^{2}$ with a pixel size of $0.855 \AA$.

All processing steps were carried out in cryoSPARC V2.15 software. All 8140 movies were motion-corrected using patch motion correction. Following patch CTF estimation, blob picking was performed using an elliptical template of size $50 \times 300 \AA$ Å. Only particles with local NCC values between 192 and 521 were kept, resulting in 3,156,357 particles that were extracted using a box size of 512 pixels and binned by 4 . Four rounds of $2 \mathrm{D}$ classification were performed to remove debris and broken particles, and the remaining 453,440 particles were extracted again using a box size of 640 pixels and downsampled to 480 pixels, resulting in a pixel 
size of $1.14 \AA$. Three ab initio models were generated and used for four rounds of 3D heterogenous refinement as to bad particles. The final particle stack, containing 117,491 particles, was used for nonuniform refinement, resulting in a $4.2 \AA$ reconstruction. The map displayed a full dimer complex, with one BmdB molecule showing much better signal than the other. Next, a mask containing this arm and the entire oxidase dimer was created with Chimera and used for local refinement as nonuniform refinement, resulting in the final $3.8 \AA$ map.

Reporting summary. Further information on research design is available in the Nature Research Reporting Summary linked to this article.

\section{Data availability}

The crystallographic data (Supplementary Table 1) used in this study are available in the Protein Data Bank database under accession codes 7ly4, 7ly5, 7ly6, and 7ly7. The cryoEM models and maps (Supplementary Tables 2 and 3) in this study are available in the Protein Data Bank database under accession codes 7ly4, Electron Microscopy Data Bank under accession codes EMD-23587 and EMD-23588. Source data are provided with this paper.

Received: 25 June 2021; Accepted: 19 December 2021; Published online: 27 January 2022

\section{References}

1. Weissman, K. J. The structural biology of biosynthetic megaenzymes. Nat. Chem. Biol. 11, 660-670 (2015).

2. Walsh, C. T. Polyketide and nonribosomal peptide antibiotics: modularity and versatility. Science 303, 1805-1810 (2004).

3. Martínez-Núñez, M. A. \& López, V. E. L. Y. Nonribosomal peptides synthetases and their applications in industry. Sustain Chem. Process 4, 13 (2016).

4. Felnagle, E. A. et al. Nonribosomal peptide synthetases involved in the production of medically relevant natural products. Mol. Pharm. 5, 191-211 (2008).

5. Bills, G. et al. New insights into the echinocandins and other fungal nonribosomal peptides and peptaibiotics. Nat. Prod. Rep. 31, 1348-1375 (2014)

6. Clardy, J. \& Walsh, C. Lessons from natural molecules. Nature 432, 829-837 (2004).

7. Makarasen, A., Yoza, K. \& Isobe, M. Higher structure of cereulide, an emetic toxin from Bacillus cereus, and special comparison with valinomycin, an antibiotic from Streptomyces fulvissimus. Chem. Asian J. 4, 688-698 (2009).

8. Raymond, K. N., Dertz, E. A. \& Kim, S. S. Enterobactin: an archetype for microbial iron transport. Proc. Natl Acad. Sci. USA 100, 3584-3588 (2003).

9. Sakurai, N., Koike, K. A., Irie, Y. \& Hayashi, H. The rice culture filtrate of Bacillus cereus isolated from emetic-type food poisoning causes mitochondrial swelling in a HEp-2 cell. Microbiol. Immunol. 38, 337-343 (1994).

10. Mikami, T. et al. An improved method for detecting cytostatic toxin (emetic toxin) of Bacillus cereus and its application to food samples. FEMS Microbiol. Lett. 119, 53-57 (1994).

11. Winn, M., Fyans, J. K., Zhuo, Y. \& Micklefield, J. Recent advances in engineering nonribosomal peptide assembly lines. Nat. Prod. Rep. 33, 317-347 (2016).

12. Reimer, J. M., Haque, A. S., Tarry, M. J. \& Schmeing, T. M. Piecing together nonribosomal peptide synthesis. Curr. Opin. Struct. Biol. 49, 104-113 (2018).

13. Payne, J. A., Schoppet, M., Hansen, M. H. \& Cryle, M. J. Diversity of nature's assembly lines-recent discoveries in non-ribosomal peptide synthesis. Mol. Biosyst. 13, 9-22 (2016).

14. Schrettl, M. et al. Distinct roles for intra- and extracellular siderophores during Aspergillus fumigatus infection. PLoS Pathog. 3, 1195-1207 (2007).

15. Zhang, S. et al. Identification of cyclic depsipeptides and their dedicated synthetase from Hapsidospora irregularis. J. Nat. Prod. 80, 363-370 (2017).

16. Bloudoff, K., Fage, C. D., Marahiel, M. A. \& Schmeing, T. M. Structural and mutational analysis of the nonribosomal peptide synthetase heterocyclization domain provides insight into catalysis. Proc. Natl Acad. Sci. USA 114, 95-100 (2017).

17. Dowling, D. P. et al. Structural elements of an NRPS cyclization domain and its intermodule docking domain. Proc. Natl Acad. Sci. USA 113, 12432-12437 (2016).

18. Bloudoff, K. \& Schmeing, T. M. Structural and functional aspects of the nonribosomal peptide synthetase condensation domain superfamily: discovery, dissection and diversity. Biochim Biophys. Acta 1865, 1587-1604 (2017).

19. Weinig, S., Mahmud, T. \& Muller, R. Markerless mutations in the myxothiazol biosynthetic gene cluster: a delicate megasynthetase with a superfluous nonribosomal peptide synthetase domain. Chem. Biol. 10, 953-960 (2003).
20. Labby, K. J., Watsula, S. G. \& Garneau-Tsodikova, S. Interrupted adenylation domains: unique bifunctional enzymes involved in nonribosomal peptide biosynthesis. Nat. Prod. Rep. 32, 641-653 (2015).

21. Finking, R. \& Marahiel, M. A. Biosynthesis of nonribosomal peptides. Аnnu Rev. Microbiol. 58, 453-488 (2004).

22. Lin, S., Van Lanen, S. G. \& Shen, B. Regiospecific chlorination of (S)-betatyrosyl-S-carrier protein catalyzed by $\mathrm{SgcC} 3$ in the biosynthesis of the enediyne antitumor antibiotic C-1027. J. Am. Chem. Soc. 129, 12432-12438 (2007).

23. Wise, C. E. \& Makris, T. M. Recruitment and regulation of the non-ribosomal peptide synthetase modifying cytochrome $\mathrm{P} 450$ involved in nikkomycin biosynthesis. ACS Chem. Biol. 12, 1316-1326 (2017).

24. Cryle, M. J., Meinhart, A. \& Schlichting, I. Structural characterization of OxyD, a cytochrome P450 involved in beta-hydroxytyrosine formation in vancomycin biosynthesis. J. Biol. Chem. 285, 24562-24574 (2010).

25. Reimmann, C. et al. Essential PchG-dependent reduction in pyochelin biosynthesis of Pseudomonas aeruginosa. J. Bacteriol. 183, 813-820 (2001).

26. Dorrestein, P. C., Yeh, E., Garneau-Tsodikova, S., Kelleher, N. L. \& Walsh, C T. Dichlorination of a pyrrolyl-S-carrier protein by FADH2-dependent halogenase PltA during pyoluteorin biosynthesis. Proc. Natl Acad. Sci. USA 102, 13843-13848 (2005)

27. Sussmuth, R. D. \& Mainz, A. Nonribosomal peptide synthesis-principles and prospects. Angew. Chem. Int. Ed. Engl. 56, 3770-3821 (2017).

28. Walsh, C. T. et al. Tailoring enzymes that modify nonribosomal peptides during and after chain elongation on NRPS assembly lines. Curr. Opin. Chem. Biol. 5, 525-534 (2001).

29. Haslinger, K., Peschke, M., Brieke, C., Maximowitsch, E. \& Cryle, M. J. $\mathrm{X}$-domain of peptide synthetases recruits oxygenases crucial for glycopeptide biosynthesis. Nature 521, 105-109 (2015).

30. Awan, A. R., Shaw, W. M. \& Ellis, T. Biosynthesis of therapeutic natural products using synthetic biology. Adv. Drug Deliv. Rev. 105, 96-106 (2016).

31. Blin, K. et al. antiSMASH 5.0: Updates to the secondary metabolite genome mining pipeline. Nucleic Acids Res. 47, W81-W87 (2019).

32. Xu, F. et al. Modified substrate specificity of a methyltransferase domain by protein insertion into an adenylation domain of the bassianolide synthetase. J. Biol. Eng. 13, 65 (2019).

33. Yuwen, L. et al. The role of aromatic L-amino acid decarboxylase in bacillamide C biosynthesis by Bacillus atrophaeus C89. Sci. Rep. 3, 1753 (2013).

34. Lu, S. et al. CDD/SPARCLE: the conserved domain database in 2020. Nucleic Acids Res. 48, D265-D268 (2020).

35. Bruice, T. C. Mechanisms of flavin catalysis. Acc. Chem. Res. 13, 256-262 (1980).

36. Jeong, S. Y., Ishida, K., Ito, Y., Okada, S. \& Murakami, M. Bacillamide, a novel algicide from the marine bacterium, Bacillus sp SY-1, against the harmful dinoflagellate, Cochlodinium polykrikoides. Tetrahedron Lett. 44, 8005-8007 (2003).

37. Socha, A. M., Long, R. A. \& Rowley, D. C. Bacillamides from a hypersaline microbial mat bacterium. J. Nat. Prod. 70, 1793-1795 (2007).

38. Martinez, V. \& Davyt, D. Total syntheses of bacillamide C and neobacillamide A; revision of their absolute configurations. Tetrahedron-Asymmetr $\mathbf{2 4}$ 1572-1575 (2013)

39. Sun, X., Liu, Y., Liu, J., Gu, G. \& Du, Y. Synthesis and structural reconfirmation of bacillamide B. Org. Biomol. Chem. 13, 4271-4277 (2015).

40. Hu, J. M., Chuenchor, W. \& Rokita, S. E. A switch between one- and twoelectron chemistry of the human flavoprotein iodotyrosine deiodinase Is controlled by substrate. J. Biol. Chem. 290, 590-600 (2015).

41. Holm, L. \& Rosenstrom, P. DALI server: conservation mapping in 3D. Nucleic Acids Res. 38, W545-W549 (2010).

42. Ghilarov, D. et al. Architecture of microcin B17 synthetase: an octameric protein complex converting a ribosomally synthesized peptide into a DNA gyrase poison. Mol. Cell 73, 749-762 (2019).

43. Burkhart, B. J., Hudson, G. A., Dunbar, K. L. \& Mitchell, D. A. A prevalent peptide-binding domain guides ribosomal natural product biosynthesis. Nat. Chem. Biol. 11, 564-U556 (2015).

44. Grove, T. L. et al. Structural Insights into thioether bond formation in the biosynthesis of sactipeptides. J. Am. Chem. Soc. 139, 11734-11744 (2017).

45. Regni, C. A. et al. How the MccB bacterial ancestor of ubiquitin E1 initiates biosynthesis of the microcin C7 antibiotic. EMBO J. 28, 1953-1964 (2009).

46. Koehnke, J. et al. Structural analysis of leader peptide binding enables leaderfree cyanobactin processing. Nat. Chem. Biol. 11, 558-563 (2015).

47. Macheroux, P., Kappes, B. \& Ealick, S. E. Flavogenomics-a genomic and structural view of flavin-dependent proteins. FEBS J. 278, 2625-2634 (2011).

48. Brunger, A. T. et al. Crystallography \& NMR system: a new software suite for macromolecular structure determination. Acta Crystallogr D. Biol. Crystallogr 54, 905-921 (1998).

49. Brunger, A. T. Version 1.2 of the crystallography and NMR system. Nat. Protoc. 2, 2728-2733 (2007). 
50. Bent, A. F. et al. Structure of the cyanobactin oxidase ThcOx from Cyanothece sp PCC 7425, the first structure to be solved at Diamond Light Source beamline I23 by means of S-SAD. Acta Crystallogr D. 72, 1174-1180 (2016).

51. Tanner, J. J., Lei, B., Tu, S. C. \& Krause, K. L. Flavin reductase P: structure of a dimeric enzyme that reduces flavin. Biochemistry 35, 13531-13539 (1996).

52. Pang, B. et al. Investigation of indigoidine synthetase reveals a conserved active-site base residue of nonribosomal peptide synthetase oxidases. J. Am. Chem. Soc. 142, 10931-10935 (2020).

53. Sieber, S. A. et al. Evidence for a monomeric structure of nonribosomal peptide synthetases. Chem. Biol. 9, 997-1008 (2002).

54. Newman, A. G., Vagstad, A. L., Storm, P. A. \& Townsend, C. A. Systematic domain swaps of iterative, nonreducing polyketide synthases provide a mechanistic understanding and rationale for catalytic reprogramming. J. Am. Chem. Soc. 136, 7348-7362 (2014)

55. Rangan, V. S., Joshi, A. K. \& Smith, S. Mapping the functional topology of the animal fatty acid synthase by mutant complementation in vitro. Biochemistry 40, 10792-10799 (2001).

56. Chen, S., Xue, Y. Q., Sherman, D. H. \& Reynolds, K. A. Mechanisms of molecular recognition in the pikromycin polyketide synthase. Chem. Biol. 7, 907-918 (2000).

57. Zheng, J., Fage, C. D., Demeler, B., Hoffman, D. W. \& Keatinge-Clay, A. T. The missing linker: a dimerization motif located within polyketide synthase modules. ACS Chem. Biol. 8, 1263-1270 (2013).

58. Gokhale, R. S., Lau, J., Cane, D. E. \& Khosla, C. Functional orientation of the acyltransferase domain in a module of the erythromycin polyketide synthase. Biochemistry 37, 2524-2528 (1998).

59. Musiol-Kroll, E. M. \& Wohlleben, W. Acyltransferases as tools for polyketide synthase engineering. Antibiotics 7, 62 (2018).

60. Kao, C. M., Pieper, R., Cane, D. E. \& Khosla, C. Evidence for two catalytically independent clusters of active sites in a functional modular polyketide synthase. Biochemistry 35, 12363-12368 (1996).

61. Qiao, C., Wilson, D. J., Bennett, E. M. \& Aldrich, C. C. A mechanism-based aryl carrier protein/thiolation domain affinity probe. J. Am. Chem. Soc. 129, 6350-6351 (2007).

62. Sundlov, J. A., Shi, C., Wilson, D. J., Aldrich, C. C. \& Gulick, A. M. Structural and functional investigation of the intermolecular interaction between NRPS adenylation and carrier protein domains. Chem. Biol. 19, 188-198 (2012).

63. Bloudoff, K., Rodionov, D. \& Schmeing, T. M. Crystal structures of the first condensation domain of CDA synthetase suggest conformational changes during the synthetic cycle of nonribosomal peptide synthetases. J. Mol. Biol. 425, 3137-3150 (2013)

64. Kosol, S. et al. Structural basis for chain release from the enacyloxin polyketide synthase. Nat. Chem. 11, 913-923 (2019).

65. Alonzo, D. A., Chiche-Lapierre, C., Tarry, M. J., Wang, J. \& Schmeing, T. M. Structural basis of keto acid utilization in nonribosomal depsipeptide synthesis. Nat. Chem. Biol. 16, 493-496 (2020).

66. Mitchell, C. A., Shi, C., Aldrich, C. C. \& Gulick, A. M. Structure of PA1221, a nonribosomal peptide synthetase containing adenylation and peptidyl carrier protein domains. Biochemistry 51, 3252-3263 (2012).

67. Reimer, J. M. et al. Structures of a dimodular nonribosomal peptide synthetase reveal conformational flexibility. Science 366, 706 (2019).

68. Chen, J. M., Sawyer, N. \& Regan, L. Protein protein interactions: general trends in the relationship between binding affinity and interfacial buried surface area. Protein Sci. 22, 510-515 (2013).

69. Xu, F., Gage, D. \& Zhan, J. Efficient production of indigoidine in Escherichia coli. J. Ind. Microbiol. Biotechnol. 42, 1149-1155 (2015).

70. Hur, G. H., Vickery, C. R. \& Burkart, M. D. Explorations of catalytic domains in non-ribosomal peptide synthetase enzymology. Nat. Prod. Rep. 29, 1074-1098 (2012).

71. Keatinge-Clay, A. T. The structures of type I polyketide synthases. Nat. Prod. Rep. 29, 1050-1073 (2012).

72. Hillson, N. J., Balibar, C. J. \& Walsh, C. T. Catalytically inactive condensation domain $\mathrm{C} 1$ is responsible for the dimerization of the VibF subunit of vibriobactin synthetase. Biochemistry 43, 11344-11351 (2004).

73. Katsuyama, Y. et al. Structural and functional analyses of the tridomainnonribosomal peptide synthetase FmoA3 for 4-methyloxazoline ring formation. Angew. Chem. Int. Ed. Engl. 60, 14554-14562 (2021).

74. Tanovic, A., Samel, S. A., Essen, L. O. \& Marahiel, M. A. Crystal structure of the termination module of a nonribosomal peptide synthetase. Science 321, 659-663 (2008).

75. Miller, B. R., Drake, E. J., Shi, C., Aldrich, C. C. \& Gulick, A. M. Structures of a nonribosomal peptide synthetase module bound to $\mathrm{MbtH}$-like proteins support a highly dynamic domain architecture. J. Biol. Chem. 291, 22559-22571 (2016).

76. Kreitler, D. F., Gemmell, E. M., Schaffer, J. E., Wencewicz, T. A. \& Gulick, A. $\mathrm{M}$. The structural basis of $\mathrm{N}$-acyl-alpha-amino-beta-lactone formation catalyzed by a nonribosomal peptide synthetase. Nat. Commun. 10, 3432 (2019).
77. Tanner, J., Lei, B. F., Liu, M. Y., Tu, S. C. \& Krause, K. L. Crystallization and preliminary crystallographic analysis of NADPH-FMN oxidoreductase from Vibrio harveyi. J. Mol. Biol. 241, 283-287 (1994).

78. Kobori, T. et al. Structure and site-directed mutagenesis of a flavoprotein from Escherichia coli that reduces nitrocompounds-alteration of pyridine nucleotide binding by a single amino acid substitution. J. Biol. Chem. 276, 2816-2823 (2001)

79. Haslinger, K. et al. The structure of a transient complex of a nonribosomal peptide synthetase and a cytochrome P450 monooxygenase. Angew. Chem. Int Ed. Engl. 53, 8518-8522 (2014).

80. Thapa, H. R., Robbins, J. M., Moore, B. S. \& Agarwal, V. Insights into thiotemplated pyrrole biosynthesis gained from the crystal structure of flavindependent oxidase in complex with carrier protein. Biochemistry 58, 918-929 (2019).

81. Schneider, T. L., Shen, B. \& Walsh, C. T. Oxidase domains in epothilone and bleomycin biosynthesis: thiazoline to thiazole oxidation during chain elongation. Biochemistry 42, 9722-9730 (2003).

82. Du, L., Chen, M., Sanchez, C. \& Shen, B. An oxidation domain in the BlmIII non-ribosomal peptide synthetase probably catalyzing thiazole formation in the biosynthesis of the anti-tumor drug bleomycin in Streptomyces verticillus ATCC15003. FEMS Microbiol Lett. 189, 171-175 (2000).

83. Silakowski, B. et al. New lessons for combinatorial biosynthesis from myxobacteria. The myxothiazol biosynthetic gene cluster of Stigmatella aurantiaca DW4/3-1. J. Biol. Chem. 274, 37391-37399 (1999).

84. Du, L., Chen, M., Zhang, Y. \& Shen, B. BlmIII and BlmIV nonribosomal peptide synthetase-catalyzed biosynthesis of the bleomycin bithiazole moiety involving both in cis and in trans aminoacylation. Biochemistry 42, 9731-9740 (2003).

85. Beer, R. et al. Creating functional engineered variants of the single-module non-ribosomal peptide synthetase IndC by T domain exchange. Mol. Biosyst. 10, 1709-1718 (2014)

86. Vickery, C. R. et al. Dissecting modular synthases through inhibition: a complementary chemical and genetic approach. Bioorg. Med Chem. Lett. 30 $126820(2020)$

87. Mori, S. et al. Structural basis for backbone $\mathrm{N}$-methylation by an interrupted adenylation domain. Nat. Chem. Biol. 14, 428-430 (2018).

88. Yang, J. Y. et al. Improved protein structure prediction using predicted interresidue orientations. Proc. Natl Acad. Sci. USA 117, 1496-1503 (2020).

89. Whicher, J. R. et al. Structural rearrangements of a polyketide synthase module during its catalytic cycle. Nature 510, 560-564 (2014).

90. Lomakin, I. B., Xiong, Y. \& Steitz, T. A. The crystal structure of yeast fatty acid synthase, a cellular machine with eight active sites working together. Cell 129, 319-332 (2007).

91. Pfeifer, B. A., Admiraal, S. J., Gramajo, H., Cane, D. E. \& Khosla, C Biosynthesis of complex polyketides in a metabolically engineered strain of $E$. coli. Science 291, 1790-1792 (2001)

92. Duerfahrt, T., Eppelmann, K., Muller, R. \& Marahiel, M. A. Rational design of a bimodular model system for the investigation of heterocyclization in nonribosomal peptide biosynthesis. Chem. Biol. 11, 261-271 (2004).

93. Leslie, A. G. W. \& Powell, H. R. Processing diffraction data with MOSFLM. Evol. Methods Macromol. Crystallogr. 245, 41-51 (2007).

94. Evans, P. R. \& Murshudov, G. N. How good are my data and what is the resolution? Acta Crystallogr D. Biol. Crystallogr 69, 1204-1214 (2013).

95. Vagin, A. \& Lebedev, A. MoRDa, an automatic molecular replacement pipeline. Acta Crystallogr A 71, S19-S19 (2015).

96. Adams, P. D. et al. PHENIX: a comprehensive Python-based system for macromolecular structure solution. Acta Crystallogr D. Biol. Crystallogr 66 213-221 (2010).

97. Emsley, P., Lohkamp, B., Scott, W. G. \& Cowtan, K. Features and development of Coot. Acta Crystallogr D. Biol. Crystallogr 66, 486-501 (2010)

98. Jhulki, I., Chanani, P. K., Abdelwahed, S. H. \& Begley, T. P. A remarkable oxidative cascade that replaces the riboflavin C8 methyl with an amino group during roseoflavin biosynthesis. J. Am. Chem. Soc. 138, 8324-8327 (2016).

99. Nazi, I., Koteva, K. P. \& Wright, G. D. One-pot chemoenzymatic preparation of coenzyme A analogues. Anal. Biochem. 324, 100-105 (2004).

100. Otwinowski, Z. \& Minor, W. Processing of X-ray diffraction data collected in oscillation mode. Methods Enzymol. 276, 307-326 (1997).

101. Waterhouse, A. et al. SWISS-MODEL: homology modelling of protein structures and complexes. Nucleic Acids Res. 46, W296-W303 (2018).

102. Chalut, C., Botella, L., de Sousa-D’Auria, C., Houssin, C. \& Guilhot, C. The nonredundant roles of two 4'-phosphopantetheinyl transferases in vital processes of Mycobacteria. Proc. Natl Acad. Sci. USA 103, 8511-8516 (2006).

103. Kong, L. et al. Divergent biosynthesis of C-nucleoside minimycin and indigoidine in bacteria. iScience 22, 430-440 (2019).

104. Mastronarde, D. N. Automated electron microscope tomography using robust prediction of specimen movements. J. Struct. Biol. 152, 36-51 (2005). 


\section{Acknowledgements}

We thank all the members of the Schmeing lab for important advice and ongoing discussions on this project; David Dai for help with cloning; synchrotron staff Shaun Labiuk (Canadian Light Source), and Frank Murphy and Surajit Banerjee (Advanced Photon Source) for facilitating remote collection of diffraction datasets; and staff at the McGill University Facility for EM Research for help with EM data collection. Special thanks go to Dr. Christopher Thibodeaux for many invaluable discussions and his expertise in flavin-dependent enzymes and RiPPs, to Dr. Martin Weigt and Dr. Juan Rodriguez-Rivas at Sorbonne Université for co-evolution discussions and to Nancy Rogerson for proofreading. We are grateful for small molecules from John Colucci and Robert Zamboni (Zamboni Chem Solution) and a gift of small molecules from Jon Patteson and Dr. Bo Li (University of North Carolina Chapel Hill). We thank Christian Chalut (CR-CNRS) for the generous gift of E. coli BL21 (DE3) entD-. This work includes research conducted at the Northeastern Collaborative Access Team beamlines, which are funded by the National Institute of General Medical Sciences from the National Institutes of Health (P30 GM124165). The Eiger $16 \mathrm{M}$ detector on the 24-ID-E beamline is funded by a NIH-ORIP HEI grant (S10OD021527). This research used resources of the Advanced Photon Source, a U.S. Department of Energy (DOE) Office of Science User Facility operated for the DOE Office of Science by Argonne National Laboratory under Contract No. DE-AC02-06CH11357. Data were collected using beamline 08-ID-1 at the Canadian Light Source, a national research facility at the University of Saskatchewan which is supported by the Canada Foundation for Innovation (CFI), the Natural Sciences and Engineering Research Council (NSERC), the National Research Council (NRC), the Canadian Institutes of Health Research (CIHR), the Government of Saskatchewan, and the University of Saskatchewan. This work was funded by CIHR (FDN-148472, 178084) Grants and a Canada Research Chair to TMS, and NSERC MSc and PhD awards to CMF.

\section{Author contributions}

T.M.S. and C.M.F. designed the study and wrote the manuscript. C.M.F. performed biochemical experiments with help from C.H. C.M.F. performed the crystallography. C.M.F., K.B., and T.M.S. determined the structure of BmdC. C.M.F. determined the cocomplex crystal structures, with help in refinement from I.S. I.S. and M.S. performed the electron microscopy, data analyses, and map calculations.

\section{Competing interests}

The authors declare no competing interests.

\section{Additional information}

Supplementary information The online version contains supplementary material available at https://doi.org/10.1038/s41467-022-28221-y.

Correspondence and requests for materials should be addressed to T. Martin Schmeing.

Peer review information Nature Communications thanks Zhiyong Li and the other anonymous reviewers for their contribution to the peer review of this work. Peer reviewer reports are available.

Reprints and permission information is available at http://www.nature.com/reprints

Publisher's note Springer Nature remains neutral with regard to jurisdictional claims in published maps and institutional affiliations.

\section{(c) (i)}

Open Access This article is licensed under a Creative Commons Attribution 4.0 International License, which permits use, sharing, adaptation, distribution and reproduction in any medium or format, as long as you give appropriate credit to the original author(s) and the source, provide a link to the Creative Commons license, and indicate if changes were made. The images or other third party material in this article are included in the article's Creative Commons license, unless indicated otherwise in a credit line to the material. If material is not included in the article's Creative Commons license and your intended use is not permitted by statutory regulation or exceeds the permitted use, you will need to obtain permission directly from the copyright holder. To view a copy of this license, visit http://creativecommons.org/ licenses/by/4.0/.

(C) The Author(s) 2022 